\title{
ON THE CONTINUATION OF SOLUTIONS FOR ELLIPTIC EQUATIONS IN TWO VARIABLES
}

\author{
SUR LE PROLONGEMENT DES SOLUTIONS DES \\ ÉQUATIONS ELLIPTIQUES DE DEUX VARIABLES
}

\author{
Frank MÜLLER \\ Institut für Mathematik der Brandenburgischen Technischen Universität, Universitätsplatz, 3-4, \\ 03044 Cottbus, Germany
}

Received 10 October 2001, revised 1 November 2001

\begin{abstract}
We consider nonlinear elliptic differential equations of second order in two variables $F\left(x, y, z(x, y), z_{x}(x, y), \ldots, z_{y y}(x, y)\right)=0,(x, y) \in \Omega \subset \mathbb{R}^{2}$. Supposing analyticity of $F$, we prove analyticity of the real solution $z=z(x, y)$ in the open set $\Omega$. Furthermore, we show that $z$ may be continued as a real analytic solution for $F=0$ across the real analytic boundary arc $\Gamma \subset \partial \Omega$, if $z$ satisfies one of the boundary conditions $z=\varphi$ or $z_{\mathbf{n}}=\psi\left(x, y, z, z_{\mathbf{t}}\right)$ on $\Gamma$ with real analytic functions $\varphi$ and $\psi$, respectively $\left(z_{\mathbf{n}}\right.$ denotes the derivative of $z$ w.r.t. the outer normal $\mathbf{n}$ on $\Gamma$ and $z_{\mathbf{t}}$ its derivative w.r.t. the tangent). The proof is based on ideas of $\mathrm{H}$. Lewy combined with a uniformization method. Studying quasilinear equations, we get somewhat better results concerning the initial regularity of the given solution and a little more insight.

(C) 2002 L'Association Publications de l'Institut Henri Poincaré. Published by Elsevier B.V. All rights reserved
\end{abstract}

RÉSUMÉ. - Nous considérons les équations différentielles non-linéaires elliptiques d'ordre deuxième dépendant de deux variables $F\left(x, y, z(x, y), z_{x}(x, y), \ldots, z_{y y}(x, y)\right)=0,(x, y) \in$ $\Omega \subset \mathbb{R}^{2}$. Supposent l'analyticité de $F$, nous démontrons l'analyticité de la solution réelle $z=z(x, y)$ dans l'ensemble ouvert $\Omega$. En outre, nous démontrons qu' on peut prolonger $z$ comme solution analytique réelle de $F=0$ à travers la courbe $\Gamma \subset \partial \Omega$, si $z$ vérifie une des conditions aux limites $z=\varphi$ or $z_{\mathbf{n}}=\psi\left(x, y, z, z_{\mathbf{t}}\right)$ sur $\Gamma$ avec des fonctions analytiques réelles $\varphi$ et $\psi\left(z_{\mathbf{n}}\right.$ designe la dérivée de $z$ par rapport à la normale extérieure $\mathbf{n}$ sur $\Gamma$ et $z_{\mathbf{t}}$ la dérivée de $z$ par rapport à la tangente). La démonstration est fondée sur des idées de H. Lewy, combinées avec une méthode d'uniformisation. En regardant des équations quasi-linéaires, nous réalisons des résultats améliorés en ce qui concerne la regularité initiale de la solution donnée et un peu plus de compréhension.

(C) 2002 L'Association Publications de l'Institut Henri Poincaré. Published by Elsevier B.V. All rights reserved

AMS classification: 35B60; 35J60; 35A30

E-mail address: mueller@math.tu-cottbus.de (F. Müller). 


\section{Introduction}

Let $z=z(x, y)$ be a solution of the fully nonlinear equation

$$
F\left(x, y, z(x, y), z_{x}(x, y), z_{y}(x, y), z_{x x}(x, y), z_{x y}(x, y), z_{y y}(x, y)\right)=0 \quad \text { in } \Omega,
$$

which is supposed to be elliptic w.r.t. $z$. Moreover, let $\Gamma \subset \partial \Omega$ be an open boundary arc of the open set $\Omega \subset \mathbb{R}^{2}$, and either the Dirichlet condition

$$
z(x, y)=\varphi(x, y), \quad(x, y) \in \Gamma,
$$

or the nontangential boundary condition of first order

$$
z_{\mathbf{n}}(x, y)=\psi\left(x, y, z(x, y), z_{\mathbf{t}}(x, y)\right), \quad(x, y) \in \Gamma,
$$

is satisfied $\left(z_{\mathbf{n}}\right.$ denotes the derivative of $z$ w.r.t. the outer normal $\mathbf{n}, z_{\mathbf{t}}$ its derivative w.r.t. the tangent $\mathbf{t}$ ).

Then we show: If $F$ is a real analytic function and $z \in C^{2}(\Omega)$, then $z$ is real analytic in $\Omega$ (Theorem 3). Furthermore, supposing analyticity of all data $F, \varphi$ or $\psi$, and $\Gamma$, we can continue $z \in C^{2}(\Omega \cup \Gamma)$ across $\Gamma$ as a real analytic solution of (1.1) (Theorem 4).

The first result is Bernstein's analyticity theorem (compare e.g. [10]; for further references cf. [12] §5.8). The second statement follows also from Morrey's far reaching Theorem 6.8.2 in [12], but its proof is quite complex. (For similar results cf. A. Friedman's paper [3].) Therefore, it seems desirable to find a more elementary and geometric proof for the equations considered here. Furthermore, our method is constructive, and one may estimate the domain of existence for the extended solution, quantifying the fundamental Theorem 2 in [13].

In this theorem we have extended a given solution of an analytic system

$$
\Delta \mathbf{z}(u, v)=\mathbf{h}\left(\mathbf{z}(u, v), \mathbf{z}_{u}(u, v), \mathbf{z}_{v}(u, v)\right),
$$

across a straight part of the boundary, where certain analytic mixed boundary conditions are satisfied. Now, introducing isothermal parameters, we transform the equation (1.1) to such a system for the vector-valued function $\mathbf{z}$ with the components: $x, y$, the solution $z$, and its first and second derivatives, all considered as functions of the isothermal parameters $(u, v)$ (cf. Lemma 3 ). Additionally, we obtain a system of first order, which we use in connection with (1.2) or (1.3) to derive suitable mixed boundary conditions for $\mathbf{z}$. Exploiting Theorems 2 and 3 of [13], we infer the analyticity of $\mathbf{z}$ and construct a continuation of $\mathbf{z}$ across the boundary. Using E. Hopf's maximum principle, we obtain the analogous results for $z=z(x, y)$ (cf. Theorems 3 and 4).

In the case of quasilinear equations

$$
a\left(x, y, z(x, y), z_{x}(x, y), z_{y}(x, y)\right) z_{x x}+2 b(\ldots) z_{x y}+c(\ldots) z_{y y}+d(\ldots)=0 \quad \text { in } \Omega
$$

the same method as above gives somewhat more insight and also better results, since the system (1.4) contains the first derivatives of $z$ only (cf. Lemma 1). Therefore, we 
dedicate the first part of the paper to equation (1.5). We find an analytic continuation of the solution $z$, even if we only suppose $z \in C^{2}(\Omega) \cap C^{1}(\Omega \cup \Gamma$ ) (Theorem 2).

In order to obtain the optimal results concerning the initial regularity for $z$, we have to combine our method with a lemma of Heinz [7] following the ideas of [8]. Regarding the optimality we refer the reader to the Examples 2 and 3.

Our method rests on H. Lewy's idea [10] of continuing a solution for (1.1) to complexvalued variables $x, y$ by solving hyperbolic initial value problems. The transition to the system (1.2) simplifies this method, since the corresponding hyperbolic problems have straight characteristic lines. In addition, we are able to continue a solution of (1.2) across the boundary. This continuation process is described in [13].

We would like to mention P. Garabedian's beautiful book [4], which was highly inspiring for us.

This paper is part of my doctoral thesis which has been accepted by the Fakultät für Mathematik, Naturwissenschaften und Informatik at the Brandenburgische Technische Universität Cottbus in November 2000.

Finally, I would like to thank Prof. Friedrich Sauvigny for his interest in my work and for many stimulating lectures and discussions. I am also very grateful to Prof. Stefan Hildebrandt for his generous support.

\section{Reduction of quasilinear equations to the normal form}

Let the functions

$$
\begin{aligned}
& a=a(x, y, z, p, q), \quad b=b(x, y, z, p, q), \\
& c=c(x, y, z, p, q), \quad d=d(x, y, z, p, q), \\
& a, b, c, d: \Theta \rightarrow \mathbb{R} \in C^{1+\alpha}(\Theta), \quad \alpha \in(0,1),
\end{aligned}
$$

be defined on an open set $\Theta \subset \mathbb{R}^{5}$. We consider a solution $z=z(x, y): \Omega \rightarrow \mathbb{R} \in C^{2}(\Omega)$ of the quasilinear differential equation

$a\left(x, y, z(x, y), z_{x}(x, y), z_{y}(x, y)\right) z_{x x}+b(\ldots) z_{x y}+c(\ldots) z_{y y}+d(\ldots)=0, \quad(x, y) \in \Omega$,

which is supposed to be elliptic with respect to $z$, i.e.

$$
\Sigma_{(x, y, z(x, y), z x(x, y), z y(x, y))}:=a c-\left.b^{2}\right|_{\left(x, y, z(x, y), z_{x}(x, y), z_{y}(x, y)\right)}>0 \quad \text { for all } \quad(x, y) \in \Omega .
$$

Obviously, we have to assume $\left\{\left(x, y, z(x, y), z_{x}(x, y), z_{y}(x, y)\right) \mid(x, y) \in \Omega\right\} \subset \Theta$. We use the abbreviations

$$
p=z_{x}, \quad q=z_{y}, \quad r=z_{x x}, \quad s=z_{x y}, \quad t=z_{y y}
$$

for the derivatives of $z$. Finally, for arbitrary $\left(x_{0}, y_{0}\right) \in \mathbb{R}^{2}$ and $r>0$ we set $B_{r}\left(x_{0}, y_{0}\right):=$ $\left\{(x, y) \in \mathbb{R}^{2} \mid\left(x-x_{0}\right)^{2}+\left(y-y_{0}\right)^{2}<r^{2}\right\}$ and write $B:=B_{1}(0,0)$. 
Lemma 1. - Let $z(x, y): \Omega \rightarrow \mathbb{R} \in C^{2}(\Omega)$ be a solution of the quasilinear equation (2.2), which is supposed to be elliptic with respect to $z$ in the sense of (2.3). Then, for any $\left(x_{0}, y_{0}\right) \in \Omega$ and any $r>0$ with $B_{r}\left(x_{0}, y_{0}\right) \subset \subset \Omega$ there exists a mapping

$$
f(u, v)=(x(u, v), y(u, v)): \bar{B} \rightarrow \overline{B_{r}\left(x_{0}, y_{0}\right)} \in C^{2+\alpha}(\bar{B}), \quad f(0,0)=\left(x_{0}, y_{0}\right),
$$

such that the relations

$$
\begin{aligned}
& y_{u}-\frac{b}{a} x_{u}+\frac{\sqrt{\Sigma}}{a} x_{v}=0, \\
& y_{v}-\frac{\sqrt{\Sigma}}{a} x_{u}-\frac{b}{a} x_{v}=0, \\
& p_{u}+\frac{b}{a} q_{u}+\frac{\sqrt{\Sigma}}{a} q_{v}+\frac{d}{a} x_{u}=0, \\
& p_{v}-\frac{\sqrt{\Sigma}}{a} q_{u}+\frac{b}{a} q_{v}+\frac{d}{a} x_{v}=0, \quad(u, v) \in B,
\end{aligned}
$$

are fulfilled (write $z(u, v):=z \circ f(u, v)$ etc.). Furthermore, the vector-valued function

$$
\mathbf{z}(u, v):=(x(u, v), y(u, v), z(u, v), p(u, v), q(u, v)) \in C^{2}\left(\bar{B}, \mathbb{R}^{5}\right)
$$

satisfies the semilinear system

$$
\Delta \mathbf{z}(u, v)=\mathbf{h}\left(\mathbf{z}(u, v), \mathbf{z}_{u}(u, v), \mathbf{z}_{v}(u, v)\right), \quad(u, v) \in B .
$$

The right hand side $\mathbf{h}=\mathbf{h}(\mathbf{z}, \mathbf{p}, \mathbf{q}) \in C^{\alpha}\left(\Theta_{0} \times \mathbb{R}^{5} \times \mathbb{R}^{5}, \mathbb{R}^{5}\right)$ is defined on the open set $\Theta_{0}:=\{\mathbf{z} \in \Theta \mid \Sigma(\mathbf{z})>0\} \subset \mathbb{R}^{5}$ and will be specified in the proof.

\section{Proof. -}

(1) At first, a $C^{2}$-solution for Eq. (2.2) belongs to $C^{3}(\Omega, \mathbb{R})$ since we have $a, b, c, d \in$ $C^{1+\alpha}(\Theta, \mathbb{R})$ (cf. [9] Satz 5 and [14] Theorem 1). We consider the elliptic Riemannian metric (w.l.o.g. we may assume $a>0$ )

$$
d s^{2}:=a(x, y) d y^{2}-2 b(x, y) d x d y+c(x, y) d x^{2}, \quad(x, y) \in \Omega,
$$

with $a(x, y):=a(x, y, z(x, y), p(x, y), q(x, y)) \in C^{1+\alpha}(\Omega)$ and so on. The uniformization theorem for nonanalytic metrics (compare, e.g., [15]) yields: For any $\left(x_{0}, y_{0}\right) \in \Omega$ and any $r>0$ with $B_{r}\left(x_{0}, y_{0}\right) \subset \subset \Omega$ there exists a $C^{2+\alpha}$-diffeomorphism $f(u, v)=$ $(x(u, v), y(u, v)): \bar{B} \rightarrow \overline{B_{r}\left(x_{0}, y_{0}\right)}$ such that $f(0,0)=\left(x_{0}, y_{0}\right)$ and such that the metric $d s^{2}$ appears in the form

$$
d s^{2}=\Lambda(u, v)\left(d u^{2}+d v^{2}\right) \quad \text { in } \bar{B}, \Lambda \in C^{1+\alpha}(\bar{B}, \mathbb{R}) .
$$

The surface element satisfies $\Lambda>0$ in $B$, and we have $J_{f}:=x_{u} y_{v}-x_{v} y_{u}>0$ in $B$ for the Jacobian of $f$.

Now, writing $w:=f^{-1}$ for the inverse mapping of $f$, we set $\Lambda(x, y):=\Lambda \circ w(x, y)$. From (2.7) we conclude 


$$
\begin{aligned}
d s^{2} & =\Lambda\left\{\left(u_{x} d x+u_{y} d y\right)^{2}+\left(v_{x} d x+v_{y} d y\right)^{2}\right\} \\
& =\Lambda\left\{\left(u_{x}^{2}+v_{x}^{2}\right) d x^{2}+2\left(u_{x} u_{y}+v_{x} v_{y}\right) d x d y+\left(u_{y}^{2}+v_{y}^{2}\right) d y^{2}\right\}
\end{aligned}
$$

Comparing this with (2.6), we obtain the conformality relations

$$
\begin{aligned}
& u_{x}^{2}+v_{x}^{2}=\frac{c}{\Lambda}, \\
& u_{x} u_{y}+v_{x} v_{y}=-\frac{b}{\Lambda}, \\
& u_{y}^{2}+v_{y}^{2}=\frac{a}{\Lambda}, \quad(x, y) \in B_{r}\left(x_{0}, y_{0}\right) .
\end{aligned}
$$

Denoting by $J_{w}$ the Jacobian of the map $w$ and using $J_{w}, \Lambda, \Sigma>0$, we get

$$
\begin{aligned}
\sqrt{\Sigma} u_{y} & =\sqrt{a c-b^{2}} u_{y}=\Lambda \sqrt{\left(u_{x}^{2}+v_{x}^{2}\right)\left(u_{y}^{2}+v_{y}^{2}\right)-\left(u_{x} u_{y}+v_{x} v_{y}\right)^{2}} u_{y} \\
& =\Lambda\left(u_{x} v_{y}-u_{y} v_{x}\right) u_{y}=\Lambda\left[\left(u_{x} u_{y}+v_{x} v_{y}\right) v_{y}-\left(u_{y}^{2}+v_{y}^{2}\right) v_{x}\right] \\
& =-b v_{y}-a v_{x} \quad \text { in } B_{r}\left(x_{0}, y_{0}\right)
\end{aligned}
$$

as well as

$$
\begin{aligned}
\sqrt{\Sigma} v_{y} & =\Lambda\left(u_{x} v_{y}-u_{y} v_{x}\right) v_{y}=\Lambda\left[\left(u_{y}^{2}+v_{y}^{2}\right) u_{x}-\left(u_{x} u_{y}+v_{x} v_{y}\right) u_{y}\right] \\
& =a u_{x}+b u_{y} \quad \text { in } B_{r}\left(x_{0}, y_{0}\right)
\end{aligned}
$$

Now, applying the relation

$$
\left(\begin{array}{ll}
x_{u} & x_{v} \\
y_{u} & y_{v}
\end{array}\right)=\left(\begin{array}{ll}
u_{x} & u_{y} \\
v_{x} & v_{y}
\end{array}\right)^{-1}=\frac{1}{J_{w}}\left(\begin{array}{cc}
v_{y} & -u_{y} \\
-v_{x} & u_{x}
\end{array}\right),
$$

we derive the Beltrami system for the uniformizing map $f$

$$
\begin{aligned}
& y_{u}-\frac{b}{a} x_{u}+\frac{\sqrt{\Sigma}}{a} x_{v}=0, \\
& y_{v}-\frac{\sqrt{\Sigma}}{a} x_{u}-\frac{b}{a} x_{v}=0, \quad(u, v) \in B .
\end{aligned}
$$

For later application we note the equivalent system

$$
\begin{aligned}
& x_{u}-\frac{b}{c} y_{u}-\frac{\sqrt{\Sigma}}{c} y_{v}=0, \\
& x_{v}+\frac{\sqrt{\Sigma}}{c} y_{u}-\frac{b}{c} y_{v}=0, \quad(u, v) \in B .
\end{aligned}
$$

(This derivation of Eqs. (2.10) follows the book [16] Section 6.1 by F. Schulz; we include it for the sake of completeness.)

(2) The first equations (2.10) and (2.11) together with the quasilinear equation (2.2) yield 


$$
\begin{aligned}
a p_{u} & =a z_{x x} x_{u}+a z_{x y} y_{u} \\
& =-2 b z_{x y} x_{u}-c z_{y y} x_{u}+a z_{x y} y_{u}-d x_{u} \\
& =z_{x y}\left(-2 b x_{u}+a y_{u}\right)-z_{y y}\left(c x_{u}\right)-d x_{u} \\
& =z_{y x}\left(-b x_{u}-\sqrt{\Sigma} x_{v}\right)-z_{y y}\left(b y_{u}+\sqrt{\Sigma} y_{v}\right)-d x_{u} \\
& =-b\left(q_{x} x_{u}+q_{y} y_{u}\right)-\sqrt{\Sigma}\left(q_{x} x_{v}+q_{y} y_{v}\right)-d x_{u} \\
& =-b q_{u}-\sqrt{\Sigma} q_{v}-d x_{u}, \quad(u, v) \in B,
\end{aligned}
$$

and from the second equations in (2.10) and (2.11) we infer

$$
\begin{aligned}
a p_{v} & =a z_{x x} x_{v}+a z_{x y} y_{v} \\
& =-2 b z_{x y} x_{v}-c z_{y y} x_{v}+a z_{x y} y_{v}-d x_{v} \\
& =z_{x y}\left(-2 b x_{v}+a y_{v}\right)-z_{y y}\left(c x_{v}\right)-d x_{v} \\
& =z_{y x}\left(-b x_{v}+\sqrt{\Sigma} x_{u}\right)-z_{y y}\left(-\sqrt{\Sigma} y_{u}+b y_{v}\right)-d x_{v} \\
& =\sqrt{\Sigma}\left(q_{x} x_{u}+q_{y} y_{u}\right)-b\left(q_{x} x_{v}+q_{y} y_{v}\right)-d x_{v} \\
& =\sqrt{\Sigma} q_{u}-b q_{v}-d x_{v}, \quad(u, v) \in B .
\end{aligned}
$$

We obtain

$$
\begin{aligned}
& p_{u}+\frac{b}{a} q_{u}+\frac{\sqrt{\Sigma}}{a} q_{v}+\frac{d}{a} x_{u}=0, \\
& p_{v}-\frac{\sqrt{\Sigma}}{a} q_{u}+\frac{b}{a} q_{v}+\frac{d}{a} x_{v}=0, \quad(u, v) \in B,
\end{aligned}
$$

and collecting Eqs. (2.10), (2.12), we have established the system (2.4).

(3) It remains to show that $\mathbf{z}=(x, y, z, p, q)$ solves the system (2.5): From (2.10) we deduce

$$
\begin{aligned}
0 & =\frac{\partial}{\partial v}\left(y_{u}-\frac{b}{a} x_{u}+\frac{\sqrt{\Sigma}}{a} x_{v}\right)-\frac{\partial}{\partial u}\left(y_{v}-\frac{\sqrt{\Sigma}}{a} x_{u}-\frac{b}{a} x_{v}\right) \\
& =\frac{\sqrt{\Sigma}}{a}\left(x_{u u}+x_{v v}\right)-\left(\frac{b}{a}\right)_{v} x_{u}+\left(\frac{\sqrt{\Sigma}}{a}\right)_{v} x_{v}+\left(\frac{\sqrt{\Sigma}}{a}\right)_{u} x_{u}+\left(\frac{b}{a}\right)_{u} x_{v}
\end{aligned}
$$

and hence

$$
\frac{\sqrt{\Sigma}}{a} \Delta x=-\nabla_{\mathbf{z}}\left(\frac{b}{a}\right) \cdot\left(x_{v} \mathbf{z}_{u}-x_{u} \mathbf{z}_{v}\right)-\nabla_{\mathbf{z}}\left(\frac{\sqrt{\Sigma}}{a}\right) \cdot\left(x_{u} \mathbf{z}_{u}+x_{v} \mathbf{z}_{v}\right) \text { in } B,
$$

writing $\nabla_{\mathbf{z}}:=\left(\frac{\partial}{\partial x}, \frac{\partial}{\partial y}, \frac{\partial}{\partial z}, \frac{\partial}{\partial p}, \frac{\partial}{\partial q}\right)$. Moreover, we compute

$$
\begin{aligned}
0 & =\frac{\partial}{\partial u}\left(y_{u}-\frac{b}{a} x_{u}+\frac{\sqrt{\Sigma}}{a} x_{v}\right)+\frac{\partial}{\partial v}\left(y_{v}-\frac{\sqrt{\Sigma}}{a} x_{u}-\frac{b}{a} x_{v}\right) \\
& =\left(y_{u u}+y_{v v}\right)-\frac{b}{a}\left(x_{u u}+x_{v v}\right)-\left(\frac{b}{a}\right)_{u} x_{u}+\left(\frac{\sqrt{\Sigma}}{a}\right)_{u} x_{v}-\left(\frac{\sqrt{\Sigma}}{a}\right)_{v} x_{u}-\left(\frac{b}{a}\right)_{v} x_{v}
\end{aligned}
$$


and consequently

$$
\frac{b}{a} \Delta x-\Delta y=-\nabla_{\mathbf{z}}\left(\frac{b}{a}\right) \cdot\left(x_{u} \mathbf{z}_{u}+x_{v} \mathbf{z}_{v}\right)+\nabla_{\mathbf{z}}\left(\frac{\sqrt{\Sigma}}{a}\right) \cdot\left(x_{v} \mathbf{z}_{u}-x_{u} \mathbf{z}_{v}\right) \text { in } B .
$$

If we treat Eqs. (2.12) in the same way, we obtain

$$
\begin{aligned}
\frac{d}{a} \Delta x+\Delta p+\frac{b}{a} \Delta q= & -\nabla_{\mathbf{z}}\left(\frac{b}{a}\right) \cdot\left(q_{u} \mathbf{z}_{u}+q_{v} \mathbf{z}_{v}\right)-\nabla_{\mathbf{z}}\left(\frac{\sqrt{\Sigma}}{a}\right) \cdot\left(q_{v} \mathbf{z}_{u}-q_{u} \mathbf{z}_{v}\right) \\
& -\nabla_{\mathbf{z}}\left(\frac{d}{a}\right) \cdot\left(x_{u} \mathbf{z}_{u}+x_{v} \mathbf{z}_{v}\right)
\end{aligned}
$$

and

$$
\begin{aligned}
\frac{\sqrt{\Sigma}}{a} \Delta q= & -\nabla_{\mathbf{z}}\left(\frac{b}{a}\right) \cdot\left(q_{v} \mathbf{z}_{u}-q_{u} \mathbf{z}_{v}\right)-\nabla_{\mathbf{z}}\left(\frac{\sqrt{\Sigma}}{a}\right) \cdot\left(q_{u} \mathbf{z}_{u}+q_{v} \mathbf{z}_{v}\right) \\
& +\nabla_{\mathbf{z}}\left(\frac{d}{a}\right) \cdot\left(x_{v} \mathbf{z}_{u}-x_{u} \mathbf{z}_{v}\right)
\end{aligned}
$$

in $B$. From the integrability conditions $z_{u}=p x_{u}+q y_{u}$ and $z_{v}=p x_{v}+q y_{v}$ we get immediately

$$
0=\frac{\partial}{\partial u}\left(z_{u}-p x_{u}-q y_{u}\right)+\frac{\partial}{\partial v}\left(z_{v}-p x_{v}-q y_{v}\right),
$$

which we may write as

$$
-p \Delta x-q \Delta y+\Delta z=p_{u} x_{u}+q_{u} y_{u}+p_{v} x_{v}+q_{v} y_{v}, \quad(u, v) \in B .
$$

Collecting Eqs. (2.13)-(2.17), we have established a linear system for the unknowns $\Delta x, \ldots, \Delta q$. Since we compute

$$
\frac{1}{a^{4}}\left|\begin{array}{ccccc}
\sqrt{\Sigma} & 0 & 0 & 0 & 0 \\
b & -a & 0 & 0 & 0 \\
d & 0 & 0 & a & b \\
0 & 0 & 0 & 0 & \sqrt{\Sigma} \\
-p & -q & 1 & 0 & 0
\end{array}\right|=-\frac{\Sigma}{a^{2}}<0
$$

for the determinant of the matrix of coefficients, we may solve this system. We consider the right hand sides of Eqs. (2.13)-(2.17) as functions of $\mathbf{z}, \mathbf{z}_{u}$ and $\mathbf{z}_{v}$ and denote them by $m_{1}, \ldots, m_{5}$, respectively. Then we find

$$
\begin{aligned}
& \Delta x=\frac{a}{\sqrt{\Sigma}} m_{1}, \quad \Delta y=\frac{b}{\sqrt{\Sigma}} m_{1}-m_{2}, \\
& \Delta z=\frac{1}{\sqrt{\Sigma}}(p a+q b) m_{1}-q m_{2}+m_{5}, \\
& \Delta p=-\frac{d}{\sqrt{\Sigma}} m_{1}+m_{3}-\frac{b}{\sqrt{\Sigma}} m_{4}, \\
& \Delta q=\frac{a}{\sqrt{\Sigma}} m_{4} \quad \text { in } B .
\end{aligned}
$$


Remarks. -

(1) The coordinates $(u, v) \in \bar{B}$ are called isothermal parameters for the Riemannian metric $d s^{2}$.

(2) If the coefficients $a, b, c, d$ are real analytic in $\Theta$, the right hand side $\mathbf{h}=$ $\mathbf{h}(\mathbf{z}, \mathbf{p}, \mathbf{q})$ of the system (2.5) is a real analytic function in the open set $\Theta_{0} \times$ $\mathbb{R}^{5} \times \mathbb{R}^{5}$.

\section{Analyticity and continuation of solutions for quasilinear equations}

First we show analyticity in the interior of the domain.

THEOREM 1. - Let $\Omega \subset \mathbb{R}^{2}$ and $\Theta \subset \mathbb{R}^{5}$ be open sets. We consider a solution $z=z(x, y) \in C^{2}(\Omega, \mathbb{R})$ of the quasilinear equation

$a\left(x, y, z(x, y), z_{x}(x, y), z_{y}(x, y)\right) z_{x x}+2 b(\ldots) z_{x y}+c(\ldots) z_{y y}+d(\ldots)=0, \quad(x, y) \in \Omega$,

with $\left\{\left(x, y, z(x, y), z_{x}(x, y), z_{y}(x, y)\right) \mid(x, y) \in \Omega\right\} \subset \Theta$. The functions $a, b, c, d: \Theta \rightarrow$ $\mathbb{R}$ are supposed to be real analytic functions, and the ellipticity condition (2.3) is fulfilled. Then $z$ is real analytic in $\Omega$.

Proof. - We choose $\left(x_{0}, y_{0}\right) \in \Omega$ and $r>0$ such that the inclusion $B_{r}\left(x_{0}, y_{0}\right) \subset \subset \Omega$ is satisfied. According to Lemma 1 we may introduce isothermal parameters $(u, v) \in \bar{B}$ such that $\mathbf{z}(u, v)=(x(u, v), y(u, v), z(u, v), p(u, v), q(u, v)) \in C^{2}\left(\bar{B}, \mathbb{R}^{5}\right)$ solves the system

$$
\Delta \mathbf{z}(u, v)=\mathbf{h}\left(\mathbf{z}(u, v), \mathbf{z}_{u}(u, v), \mathbf{z}_{v}(u, v)\right), \quad(u, v) \in B,
$$

with a real analytic right hand side $\mathbf{h}: \Theta_{0} \times \mathbb{R}^{5} \times \mathbb{R}^{5} \rightarrow \mathbb{R}^{5}$ (cf. the second remark at the end of Section 2). As shown in [13] Theorem 4, any solution $\mathbf{z}$ of such a system must be real analytic in $B$. In particular, the uniformizing map $f(u, v)=(x(u, v), y(u, v))$ : $\bar{B} \rightarrow \overline{B_{r}\left(x_{0}, y_{0}\right)}$ is real analytic in $B$, diffeomorphic, and we know $J_{f}>0$ in $B$. Therefore, the inverse mapping $w=f^{-1}$ and the function $z(x, y):=z \circ w(x, y)$ are real analytic in $B_{r}\left(x_{0}, y_{0}\right)$ (cf. [5] Section 1.7, where corresponding results are proven for analytic functions in more then one variable). Since the point $\left(x_{0}, y_{0}\right) \in \Omega$ was chosen arbitrarily, we conclude the analyticity of $z(x, y)$ in $\Omega$.

Now, we study the boundary behaviour of solutions for quasilinear elliptic equations.

Let $\Gamma \subset \partial \Omega \neq \emptyset$ be an open boundary arc of $\Omega$. For any point $\left(x_{0}, y_{0}\right) \in \Gamma$ there have to be a number $\sigma>0$ and a function $h=h(x, y) \in C^{1}\left(B_{\sigma}\left(x_{0}, y_{0}\right), \mathbb{R}\right)$ such that

$$
\left|\nabla h\left(x_{0}, y_{0}\right)\right|>0
$$

and

$$
\begin{aligned}
& \Omega \cap B_{\sigma}\left(x_{0}, y_{0}\right)=\left\{(x, y) \in B_{\sigma}\left(x_{0}, y_{0}\right) \mid h(x, y)<0\right\}, \\
& \Gamma \cap B_{\sigma}\left(x_{0}, y_{0}\right)=\left\{(x, y) \in B_{\sigma}\left(x_{0}, y_{0}\right) \mid h(x, y)=0\right\} .
\end{aligned}
$$

$\Gamma$ is called a $C^{k+\alpha}-\operatorname{arc}(k \in \mathbb{N}, \alpha \in[0,1))$ if $h \in C^{k+\alpha}\left(B_{\sigma}\left(x_{0}, y_{0}\right), \mathbb{R}\right)$ is true for any $\left(x_{0}, y_{0}\right) \in \Gamma$. If $h$ is real analytic for any $\left(x_{0}, y_{0}\right) \in \Gamma$, we say $\Gamma$ is a (real) analytic arc. 
On such a boundary arc $\Gamma$ we prescribe boundary data of two kinds:

(1) Dirichlet data: We investigate the case

$$
z(x, y)=\varphi(x, y), \quad(x, y) \in \Gamma,
$$

with a function $\varphi=\varphi(x, y): \Gamma \rightarrow \mathbb{R}$.

(2) Nontangential data of first order: A function $\psi=\psi(x, y, z, \zeta): \mathcal{U} \rightarrow \mathbb{R}$ is given on an open set $\mathcal{U} \subset \mathbb{R}^{4}$. We consider the boundary condition

$$
z_{\mathbf{n}}(x, y)=\psi\left(x, y, z(x, y), z_{\mathbf{t}}(x, y)\right), \quad(x, y) \in \Gamma .
$$

Here $z_{\mathbf{n}}$ denotes the derivative of $z$ w.r.t. the outer normal $\mathbf{n}$ and $z_{\mathbf{t}}$ its derivative in tangential direction. Obviously, we have to demand $\left\{\left(x, y, z(x, y), z_{\mathbf{t}}(x, y)\right) \mid\right.$ $(x, y) \in \Gamma\} \subset \mathcal{U}$.

In the following, we replace the ellipticity condition (2.3) by

$$
\left.\Sigma\right|_{\left(x, y, z(x, y), z_{x}(x, y), z_{y}(x, y)\right)}>0, \quad(x, y) \in \Omega \cup \Gamma,
$$

i.e. we assume that the elliptic equation (3.1) does not degenerate on $\Gamma$. We always consider solutions $z=z(x, y) \in C^{2}(\Omega, \mathbb{R}) \cap C^{1}(\Omega \cup \Gamma, \mathbb{R})$ of (3.1), and we suppose

$$
\left\{\left(x, y, z(x, y), z_{x}(x, y), z_{y}(x, y)\right) \mid(x, y) \in \Omega \cup \Gamma\right\} \subset \Theta .
$$

To start our analysis, we show that we can restrict our considerations to the case $\Omega=S:=\{(x, y) \in B \mid y>0\}$ and $\Gamma=I:=\{(x, y) \in B \mid y=0\}$, following the ideas of E. Heinz [8], who has applied this method to Monge-Ampère equations:

Let $\Gamma$ be a $C^{3+\alpha}$-arc. We may assume $\left|h_{y}\left(x_{0}, y_{0}\right)\right|>0$ due to (3.2). If $\left|h_{y}\left(x_{0}, y_{0}\right)\right|=0$ is true, we have $\left|h_{x}\left(x_{0}, y_{0}\right)\right|>0$ and one may carry out the following calculations exchanging $x$ and $y$. Now, there exists $R \in(0, \sigma]$ such that we can solve the equation $h(x, y)=0$ with respect to $y$ in $B_{R}\left(x_{0}, y_{0}\right)$. More precisely, we find a function $g=g(x) \in C^{3+\alpha}\left(\left[x_{0}-R, x_{0}+R\right], \mathbb{R}\right)$ with $g\left(x_{0}\right)=0$ such that we have the representations

$$
\begin{aligned}
& \Omega \cap B_{R}\left(x_{0}, y_{0}\right)=\left\{(x, y) \in B_{R}\left(x_{0}, y_{0}\right) \mid \vartheta\left(y_{0}+g(x)-y\right)<0\right\}, \\
& \Gamma \cap B_{R}\left(x_{0}, y_{0}\right)=\left\{(x, y) \in B_{R}\left(x_{0}, y_{0}\right) \mid y=y_{0}+g(x)\right\}
\end{aligned}
$$

where $\vartheta \in\{-1,+1\}$. We choose $R_{1} \in(0, R]$ with

$$
R_{1}<\frac{R}{1+m}, \quad m:=\max _{x \in\left[x_{0}-R, x_{0}+R\right]}\left|g^{\prime}(x)\right| .
$$

Introducing new coordinates $(\xi, \eta)$ by the definition

$$
x(\xi, \eta)=x_{0}+R_{1} \xi, \quad y(\xi, \eta)=y_{0}+g\left(x_{0}+R_{1} \xi\right)+\vartheta R_{1} \eta, \quad(\xi, \eta) \in \bar{S},
$$

we have the estimate 


$$
\begin{aligned}
\left(x-x_{0}\right)^{2}+\left(y-y_{0}\right)^{2} & =R_{1}^{2} \xi^{2}+\left(g\left(x_{0}+R_{1} \xi\right)+\vartheta R_{1} \eta\right)^{2} \\
& \leqslant R_{1}^{2}+2 m R_{1}^{2}+m^{2} R_{1}^{2}=R_{1}^{2}(1+m)^{2}<R^{2}
\end{aligned}
$$

for any $(\xi, \eta) \in \bar{B}$. Therefore, we obtain the inclusions

$$
\begin{aligned}
& \{(x(\xi, \eta), y(\xi, \eta)) \mid(\xi, \eta) \in \bar{S}\} \subset\left(\bar{\Omega} \cap B_{R}\left(x_{0}, y_{0}\right)\right), \\
& \{(x(\xi, 0), y(\xi, 0)) \mid \xi \in[-1,1]\} \subset\left(\Gamma \cap B_{R}\left(x_{0}, y_{0}\right)\right) .
\end{aligned}
$$

Let us now consider the function

$$
\tilde{z}=\tilde{z}(\xi, \eta):=z\left(x_{0}+R_{1} \xi, y_{0}+g\left(x_{0}+R_{1} \xi\right)+\vartheta R_{1} \eta\right) \in C^{2}(S) \cap C^{1}(\bar{S}),
$$

where $z \in C^{2}(\Omega) \cap C^{1}(\Omega \cup \Gamma)$ denotes a given solution for (3.1). We abbreviate $\tilde{p}=\tilde{z}_{\xi}, \ldots, \tilde{t}=\tilde{z}_{\eta \eta}$ and calculate

$$
\begin{aligned}
& \left.z_{x x}\right|_{(x(\xi, \eta), y(\xi, \eta))}=R_{1}^{-2}\left(\tilde{r}-2 \vartheta g^{\prime} \tilde{s}+\left(g^{\prime}\right)^{2} \tilde{t}\right)-g^{\prime \prime} z_{y}, \\
& \left.z_{x y}\right|_{(x(\xi, \eta), y(\xi, \eta))}=R_{1}^{-2}\left(\vartheta \tilde{s}-g^{\prime} \tilde{t}\right), \\
& \left.z_{y y}\right|_{(x(\xi, \eta), y(\xi, \eta))}=R_{1}^{-2} \tilde{t}, \quad(\xi, \eta) \in S .
\end{aligned}
$$

Inserting this into (3.1), we infer

$$
\tilde{a}(\xi, \eta, \tilde{z}, \tilde{p}, \tilde{q}) \tilde{r}+2 \tilde{b}(\ldots) \tilde{s}+\tilde{c}(\ldots) \tilde{t}+\tilde{d}(\ldots)=0, \quad(\xi, \eta) \in S,
$$

with

$$
\begin{aligned}
& \tilde{a}(\xi, \eta, \tilde{z}, \tilde{p}, \tilde{q}):=a(x, y, z, p, q), \\
& \tilde{b}(\xi, \eta, \tilde{z}, \tilde{p}, \tilde{q}):=\vartheta\left\{b(\ldots)-g^{\prime}(x) a(\ldots)\right\}, \\
& \tilde{c}(\xi, \eta, \tilde{z}, \tilde{p}, \tilde{q}):=c(\ldots)-2 b(\ldots) g^{\prime}(x)+g^{\prime}(x)^{2} a(\ldots), \\
& \tilde{d}(\xi, \eta, \tilde{z}, \tilde{p}, \tilde{q}):=R_{1}^{2}\left\{d(\ldots)-a(\ldots) g^{\prime \prime}(x) q\right\} .
\end{aligned}
$$

In (3.11) the quantities $x, y, z, p, q$ are defined by the relations

$$
\begin{aligned}
& x=x_{0}+R_{1} \xi, \\
& y=y_{0}+g\left(x_{0}+R_{1} \xi\right)+\vartheta R_{1} \eta, \\
& z=\tilde{z}, \\
& p=R_{1}^{-1} \tilde{p}-\vartheta R_{1}^{-1} g^{\prime}\left(x_{0}+R_{1} \xi\right) \tilde{q}, \\
& q=\vartheta R_{1}^{-1} \tilde{q} .
\end{aligned}
$$

A simple calculation yields

$$
\widetilde{\Sigma}(\xi, \eta, \tilde{z}(\xi, \eta), \tilde{p}(\xi, \eta), \tilde{q}(\xi, \eta)):=\tilde{a} \tilde{c}-\tilde{b}^{2}=a c-b^{2}>0 \quad \text { for }(\xi, \eta) \in \bar{S}
$$


Furthermore, we have $\tilde{a}, \tilde{b}, \tilde{c}, \tilde{d} \in C^{1+\alpha}(\widetilde{\Theta}, \mathbb{R})$ due to (3.11), (3.12) and because of $g \in C^{3+\alpha}\left(\left[x_{0}-R_{1}, x_{0}+R_{1}\right], \mathbb{R}\right)$. Here $\widetilde{\Theta} \subset \mathbb{R}^{5}$ denotes an open set with the property

$$
\{(\xi, \eta, \tilde{z}(\xi, \eta), \tilde{p}(\xi, \eta), \tilde{q}(\xi, \eta)) \mid(\xi, \eta) \in \bar{S}\} \subset \widetilde{\Theta} .
$$

Now, we examine the behaviour of the boundary conditions under the transformation (3.7).

(1) Dirichlet data: Defining

$$
\tilde{\varphi}(\xi):=\varphi(x(\xi, 0), y(\xi, 0)), \quad \xi \in[-1,1],
$$

we conclude

$$
\tilde{z}(\xi, 0)=z(x(\xi, 0), y(\xi, 0))=\tilde{\varphi}(\xi), \quad \xi \in[-1,1],
$$

from (3.8) and the boundary condition (3.4).

(2) Nontangential data of first order: We consider the function

$$
\begin{aligned}
\tilde{\psi}(\xi, \tilde{z}, \tilde{p}):= & -\frac{R_{1}}{\sqrt{1+\left(g^{\prime}(x(\xi, 0))\right)^{2}}} \\
& \times \psi\left(x(\xi, 0), y(\xi, 0), \tilde{z}, \frac{\vartheta R_{1}^{-1}}{\sqrt{1+\left(g^{\prime}(x(\xi, 0))\right)^{2}}} \tilde{p}\right) \\
& +\frac{\vartheta g^{\prime}(x(\xi, 0))}{1+\left(g^{\prime}(x(\xi, 0))\right)^{2}} \tilde{p} .
\end{aligned}
$$

Since we know (we parametrize $\Gamma$ such that $\Omega$ lies on its left hand side)

$$
\begin{aligned}
\mathbf{t}(x) & =\frac{\vartheta}{\sqrt{1+\left(g^{\prime}(x)\right)^{2}}}\left(1, g^{\prime}(x)\right), \\
\mathbf{n}(x) & =\frac{\vartheta}{\sqrt{1+\left(g^{\prime}(x)\right)^{2}}}\left(g^{\prime}(x),-1\right), \quad x \in\left[x_{0}-R_{1}, x_{0}+R_{1}\right],
\end{aligned}
$$

we may calculate

$$
\begin{aligned}
\left.z_{\mathbf{t}}(x, y)\right|_{(x(\xi, 0), y(\xi, 0))} & \\
= & \frac{\vartheta}{\sqrt{1+\left(g^{\prime}\left(x_{0}+R_{1} \xi\right)\right)^{2}}}\left\{z_{x}(x, y)+g^{\prime}(x) z_{y}(x, y)\right\}_{(x(\xi, 0), y(\xi, 0))} \\
& =\frac{\vartheta}{\sqrt{1+\left(g^{\prime}\right)^{2}}}\left\{\left(\tilde{z}_{\xi} \xi_{x}+\tilde{z}_{\eta} \eta_{x}\right)+g^{\prime}\left(\tilde{z}_{\xi} \xi_{y}+\tilde{z}_{\eta} \eta_{y}\right)\right\} \\
& =\frac{\vartheta}{\sqrt{1+\left(g^{\prime}\right)^{2}}}\left\{R_{1}^{-1} \tilde{z}_{\xi}-\vartheta R_{1}^{-1} g^{\prime} \tilde{z}_{\eta}+\vartheta R_{1}^{-1} g^{\prime} \tilde{z}_{\eta}\right\} \\
& =\frac{\vartheta R_{1}^{-1}}{\sqrt{1+\left(g^{\prime}\left(x_{0}+R_{1} \xi\right)\right)^{2}}} \tilde{z}_{\xi}(\xi, 0), \quad \xi \in[-1,1] .
\end{aligned}
$$

Moreover, we obtain 


$$
\begin{aligned}
z_{\mathbf{n}} & \left.(x, y)\right|_{(x(\xi, 0), y(\xi, 0))} \\
& =\frac{\vartheta}{\sqrt{1+\left(g^{\prime}\left(x_{0}+R_{1} \xi\right)\right)^{2}}}\left\{g^{\prime}(x) z_{x}(x, y)-z_{y}(x, y)\right\}_{(x(\xi, 0), y(\xi, 0))} \\
& =\frac{\vartheta}{\sqrt{1+\left(g^{\prime}\right)^{2}}}\left\{R_{1}^{-1} g^{\prime} \tilde{z}_{\xi}-\vartheta R_{1}^{-1}\left(g^{\prime}\right)^{2} \tilde{z}_{\eta}-\vartheta R_{1}^{-1} \tilde{z}_{\eta}\right\} \\
& =\frac{\vartheta R_{1}^{-1} g^{\prime}}{\sqrt{1+\left(g^{\prime}\right)^{2}}} \tilde{z}_{\xi}-R_{1}^{-1} \sqrt{1+\left(g^{\prime}\right)^{2}} \tilde{z}_{\eta}
\end{aligned}
$$

for the normal derivative of $z$. Together with the boundary condition we infer

$$
\begin{aligned}
\tilde{z}_{\eta}(\xi, 0)= & -\frac{R_{1}}{\sqrt{1+\left(g^{\prime}\right)^{2}}} z_{\mathbf{n}}(x(\xi, 0), y(\xi, 0))+\frac{\vartheta g^{\prime}}{1+\left(g^{\prime}\right)^{2}} \tilde{z}_{\xi}(\xi, 0) \\
= & -\frac{R_{1}}{\sqrt{1+\left(g^{\prime}\right)^{2}}} \psi\left(x(\xi, 0), y(\xi, 0), \tilde{z}(\xi, 0), \frac{\vartheta R_{1}^{-1}}{\sqrt{1+\left(g^{\prime}\right)^{2}}} \tilde{z}_{\xi}(\xi, 0)\right) \\
& +\frac{\vartheta g^{\prime}}{1+\left(g^{\prime}\right)^{2}} \tilde{z}_{\xi}(\xi, 0) \\
= & \tilde{\psi}\left(\xi, \tilde{z}(\xi, 0), \tilde{z}_{\xi}(\xi, 0)\right), \quad \xi \in[-1,1] .
\end{aligned}
$$

The function $\tilde{\psi}$ is defined on a set $\widetilde{\mathcal{U}} \subset \mathbb{R}^{3}$ with the property

$$
\left\{\left(\xi, \tilde{z}(\xi, \eta), \tilde{z}_{\xi}(\xi, \eta)\right) \mid(\xi, \eta) \in \bar{S}\right\} \subset \tilde{\mathcal{U}},
$$

choosing $R>0$ sufficiently small to ensure that the inclusion

$$
\left\{\left(x, y, z(x, y), z_{\mathbf{T}}(x, y)\right) \mid(x, y) \in \bar{\Omega} \cap B_{R}\left(x_{0}, y_{0}\right)\right\} \subset \mathcal{U}
$$

is satisfied. Here $\mathcal{U}$ is the domain of definition for $\psi$, and we have set

$$
z_{\mathbf{T}}(x, y):=\frac{\vartheta}{\sqrt{1+\left(g^{\prime}(x)\right)^{2}}}\left\{z_{x}(x, y)+g^{\prime}(x) z_{y}(x, y)\right\} .
$$

Since $z_{\mathbf{T}}(x, y)$ and $z_{\mathbf{t}}(x, y)$ coincide for $(x, y) \in \Gamma \cap B_{R}\left(x_{0}, y_{0}\right)$, such a number $R>0$ exists.

Let us summarize our results:

LEMMA 2. - We consider a solution $z=z(x, y) \in C^{2}(\Omega, \mathbb{R}) \cap C^{1}(\Omega \cup \Gamma, \mathbb{R})$ for the quasilinear equation (3.1), which is supposed to be elliptic with respect to $z$ in the sense of (3.6). On the $C^{3+\alpha}$-arc $\Gamma$ we prescribe either Dirichlet data (3.4) with $\varphi \in C^{3+\alpha}(\Gamma, \mathbb{R})$ or nontangential data of first order (3.5) with $\psi \in C^{2+\alpha}(\mathcal{U}, \mathbb{R})$.

Then, defining $\tilde{z}=\tilde{z}(\xi, \eta) \in C^{2}(S, \mathbb{R}) \cap C^{1}(\bar{S}, \mathbb{R})$ as in (3.9), this function is a solution of (3.10) with coefficients $\tilde{a}, \tilde{b}, \tilde{c}, \tilde{d} \in C^{1+\alpha}(\widetilde{\Theta}, \mathbb{R})$, where $\widetilde{\Theta} \subset \mathbb{R}^{5}$ has the property (3.14). The equation is elliptic with respect to $\tilde{z}$ in the sense of (3.13). Moreover, $\tilde{z}$ fulfils one of the boundary conditions (3.16) or (3.18) with right hand sides $\tilde{\varphi} \in$ $C^{3+\alpha}([-1,1], \mathbb{R})$ and $\tilde{\psi} \in C^{2+\alpha}(\widetilde{\mathcal{U}}, \mathbb{R})$ respectively. The open set $\widetilde{\mathcal{U}} \subset \mathbb{R}^{3}$ satisfies the inclusion (3.19). 
Finally, if the coefficients $a, b, c, d$ and the boundary data $\varphi, \psi$ are real analytic functions and if $\Gamma$ is a real analytic arc, then also the transformed quantities $\tilde{a}, \tilde{b}, \tilde{c}, \tilde{d}$ and $\tilde{\varphi}, \tilde{\psi}$ are real analytic functions.

LEMMA 3. - Let $z=z(x, y) \in C^{2}(\Omega, \mathbb{R}) \cap C^{1}(\Omega \cup \Gamma, \mathbb{R})$ be a solution of (3.1) and let the ellipticity condition (3.6) be valid. We assume $a, b, c, d \in C^{1+\alpha}(\Theta, \mathbb{R})$, and $\Gamma \subset \partial \Omega$ is an open $C^{3+\alpha}$-arc. Furthermore, $z$ satisfies one of the boundary conditions (3.4) with $\varphi \in C^{3+\alpha}(\Gamma, \mathbb{R})$ or $(3.5)$ with $\psi \in C^{2+\alpha}(\mathcal{U}, \mathbb{R})$. Then we have $z \in C^{3+\alpha}(\Omega \cup \Gamma, \mathbb{R})$.

\section{Proof. -}

(1) We mention $z \in C^{3+\alpha}(\Omega)$ and choose an arbitrary point $\left(x_{0}, y_{0}\right) \in \Gamma$. Because of Lemma 2, we may restrict our considerations to the case $\Omega=S, \Gamma=I$. First we examine the case of the Dirichlet boundary condition

$$
z(x, 0)=\varphi(x), \quad x \in[-1,1],
$$

with $\varphi \in C^{3+\alpha}([-1,1], \mathbb{R})$. There exists a function $\Phi(x, y) \in C^{3+\alpha}(\bar{S}, \mathbb{R})$ with $\Phi(x, 0)=$ $\varphi(x)$ on $\bar{I}$. Now, $\hat{z}(x, y):=z(x, y)-\Phi(x, y)$ solves a quasilinear equation with $C^{1+\alpha_{-}}$ coefficients, and this equation is elliptic with respect to $\hat{z}$ up to the boundary of $S$. Apparently, the advantage of this transformation is

$$
\hat{z}(x, 0)=0, \quad x \in[-1,1] .
$$

In the sequel, we omit the hat and assume that $z$ fulfils the homogeneous Dirichlet condition (3.20).

Let us now introduce isothermal parameters $(u, v) \in \bar{S}$ with respect to the metric

$$
d s^{2}=a d y^{2}-2 b d x d y+c d x^{2} \in C^{1+\alpha}(S) \cap C^{0}(\bar{S}) .
$$

The uniformizing map

$$
f(u, v)=(x(u, v), y(u, v)): \bar{S} \rightarrow \bar{S} \in C^{2+\alpha}(S) \cap C^{0}(\bar{S})
$$

satisfies $J_{f}=x_{u} y_{v}-x_{v} y_{u}>0$ in $S$, and we have

$$
\begin{aligned}
& y_{u}-\frac{b}{a} x_{u}+\frac{\sqrt{\Sigma}}{a} x_{v}=0, \\
& y_{v}-\frac{\sqrt{\Sigma}}{a} x_{u}-\frac{b}{a} x_{v}=0, \\
& p_{u}+\frac{b}{a} q_{u}+\frac{\sqrt{\Sigma}}{a} q_{v}+\frac{d}{a} x_{u}=0, \\
& p_{v}-\frac{\sqrt{\Sigma}}{a} q_{u}+\frac{b}{a} q_{v}+\frac{d}{a} x_{v}=0, \quad(u, v) \in S,
\end{aligned}
$$


due to Lemma $1\left(p(u, v):=z_{x}(x(u, v), y(u, v)), q(u, v):=z_{y}(x(u, v), y(u, v))\right)$. Solving these equations for $x_{u}, x_{v}, q_{u}$ and $q_{v}$, one gets the equivalent system

$$
\begin{aligned}
& x_{u}-\frac{b}{c} y_{u}-\frac{\sqrt{\Sigma}}{c} y_{v}=0, \\
& x_{v}+\frac{\sqrt{\Sigma}}{c} y_{u}-\frac{b}{c} y_{v}=0, \\
& q_{u}+\frac{b}{c} p_{u}-\frac{\sqrt{\Sigma}}{c} p_{v}+\frac{d}{c} y_{u}=0, \\
& q_{v}+\frac{\sqrt{\Sigma}}{c} p_{u}+\frac{b}{c} p_{v}+\frac{d}{c} y_{v}=0, \quad(u, v) \in S .
\end{aligned}
$$

The uniformizing map $f$ may be chosen such that

$$
\begin{aligned}
& f(-1,0)=(-1,0), \quad f(0,0)=(0,0), \quad f(1,0)=(1,0), \\
& y(u, 0)=0, \quad u \in(-1,1),
\end{aligned}
$$

is valid. The function $\mathbf{z}=(x, y, z, p, q)$ solves the system (2.18), where the quantities $m_{1}, \ldots, m_{2}$ denote the right hand sides of Eqs. (2.13)-(2.17). Therefore, we may derive the estimates

$$
|\Delta x|,|\Delta y|,|\Delta z|,|\Delta p|,|\Delta q| \leqslant C\left(|\nabla x|^{2}+|\nabla y|^{2}+|\nabla p|^{2}+|\nabla q|^{2}\right) \quad \text { in } S,
$$

applying the ellipticity property and using the integrability conditions $z_{u}=p x_{u}+q y_{u}$, $z_{v}=p x_{v}+q y_{v}$. In (3.24) $C$ denotes a non-negative constant. We will not distinguish between such constants.

The first two equations in each of the systems (3.21) and (3.22) yield

$$
\frac{\sqrt{\Sigma}}{a}|\nabla x|^{2}=J_{f}=\frac{\sqrt{\Sigma}}{c}|\nabla y|^{2} \quad \text { in } S,
$$

and we learn

$$
|\nabla q| \leqslant C(|\nabla y|+|\nabla p|) \quad \text { in } S
$$

from the last two equations in (3.22). Inserting this into (3.24), we get

$$
|\Delta x|,|\Delta y|,|\Delta z|,|\Delta p|,|\Delta q| \leqslant C\left(|\nabla y|^{2}+|\nabla p|^{2}\right) \quad \text { in } S .
$$

Let us now consider the function $\mathbf{x}:=(y(u, v), p(u, v)) \in C^{2}\left(S, \mathbb{R}^{2}\right) \cap C^{0}\left(\bar{S}, \mathbb{R}^{2}\right)$. With the aid of (3.20), (3.23) and (3.27) we get

$$
\begin{aligned}
& |\Delta \mathbf{x}(u, v)| \leqslant C|\nabla \mathbf{x}(u, v)|^{2}, \quad(u, v) \in S, \\
& \mathbf{x}(u, 0)=0, \quad u \in(-1,1) .
\end{aligned}
$$


According to the Hilfssatz in [7] there exists $\varrho \in(0,1]$ such that $\mathbf{x} \in C^{1+\mu}\left(\overline{S_{\varrho}}\right)$ holds true with an arbitrary $\mu \in(0,1)$, setting $S_{\varrho}:=S \cap B_{\varrho}(0,0)$. Therefore, we obtain

$$
f(u, v), z(u, v), p(u, v), q(u, v) \in C^{1+\mu}\left(\overline{S_{\varrho}}\right)
$$

from (3.22) and the integrability conditions. Since the function $\mathbf{z}=(x, y, z, p, q)$ is a solution of the system (2.5), we know that $\mathbf{x}=(y, p)$ is a solution for

$$
\begin{aligned}
& \Delta \mathbf{x}(u, v)=\mathbf{k}(u, v), \quad(u, v) \in S_{\varrho}, \\
& \mathbf{x}(u, 0)=0, \quad u \in(-\varrho, \varrho),
\end{aligned}
$$

with

$$
\mathbf{k}(u, v):=\left(h_{2}\left(\mathbf{z}(u, v), \mathbf{z}_{u}(u, v), \mathbf{z}_{v}(u, v)\right), h_{4}\left(\mathbf{z}(u, v), \mathbf{z}_{u}(u, v), \mathbf{z}_{v}(u, v)\right)\right) \in C^{\alpha \mu}\left(\overline{S_{\varrho}}\right),
$$

where we have written $\mathbf{h}=\left(h_{1}, h_{2}, h_{3}, h_{4}, h_{5}\right)$.

Now, some potential theory shows $\mathbf{x} \in C^{2+\alpha \mu}\left(\overline{S_{\varrho / 2}}\right)$ (compare e.g. [6], Section 4.4). This again leads to $\mathbf{z} \in C^{2+\alpha \mu}\left(\overline{S_{\varrho / 2}}\right)$, and consequently we have $\mathbf{k} \in C^{\alpha}\left(\overline{S_{\varrho / 2}}\right)$. Therefore, we even infer $\mathbf{x} \in C^{2+\alpha}\left(\overline{S_{\varrho / 4}}\right)$ from (3.29), and we finally conclude

$$
f, z, p, q \in C^{2+\alpha}\left(\overline{S_{\varrho / 4}}\right) .
$$

(2) Let us now consider the second kind of boundary conditions

$$
z_{y}(x, 0)=\psi\left(x, z(x, 0), z_{x}(x, 0)\right), \quad x \in[-1,1],
$$

with a function $\psi \in C^{2+\alpha}(\mathcal{U}, \mathbb{R})$, assuming $\left\{\left(x, z(x, y), z_{x}(x, y)\right) \mid(x, y) \in \bar{S}\right\} \subset \mathcal{U}$. We introduce the auxiliary function

$$
m(u, v):=q(u, v)-\psi(x(u, v), z(u, v), p(u, v)) \in C^{2+\alpha}(S, \mathbb{R}) \cap C^{0}(\bar{S}, \mathbb{R})
$$

and define $\mathbf{y}=(x, z, p)$ as well as $\nabla_{\mathbf{y}} \psi=\left(\psi_{x}, \psi_{z}, \psi_{p}\right)$. Then, we calculate

$$
\nabla m=\nabla q-\nabla_{\mathbf{y}} \psi(\mathbf{y}) \cdot \nabla \mathbf{y} \quad \text { in } S
$$

and

$$
\Delta m=\Delta q-\mathbf{y}_{u} \cdot \nabla_{\mathbf{y}}^{2} \psi(\mathbf{y}) \cdot \mathbf{y}_{u}-\mathbf{y}_{v} \cdot \nabla_{\mathbf{y}}^{2} \psi(\mathbf{y}) \cdot \mathbf{y}_{v}-\nabla_{\mathbf{y}} \psi(\mathbf{y}) \cdot \Delta \mathbf{y} \quad \text { in } S,
$$

where $\nabla_{\mathbf{y}}^{2} \psi$ denotes the Hessian of $\psi$. If we insert the third equation in (3.22) into the first equation of the system (3.33), we obtain

$$
m_{u}=-\left(\frac{b}{c}+\psi_{p}\right) p_{u}+\frac{\sqrt{\Sigma}}{c} p_{v}-\frac{d}{c} y_{u}-\psi_{x} x_{u}-\psi_{z} z_{u}
$$


Furthermore, the fourth equation of (3.22) and the second of (3.33) yield

$$
m_{v}=-\frac{\sqrt{\Sigma}}{c} p_{u}-\left(\frac{b}{c}+\psi_{p}\right) p_{v}-\frac{d}{c} y_{v}-\psi_{x} x_{v}-\psi_{z} z_{v} .
$$

These equations form a linear system for the unknowns $p_{u}$ and $p_{v}$ which we may solve on account of

$$
\left(\frac{b}{c}+\psi_{p}\right)^{2}+\left(\frac{\sqrt{\Sigma}}{c}\right)^{2} \geqslant \frac{\Sigma}{c^{2}}>0 \text { in } \bar{S}
$$

Since we know $\left|\nabla_{\mathbf{y}} \psi(\mathbf{y}(x, y))\right| \leqslant C$ in $\bar{S}$, we infer

$$
|\nabla p| \leqslant C(|\nabla x|+|\nabla y|+|\nabla z|+|\nabla m|) \quad \text { in } S .
$$

Combining this with the integrability conditions and (3.25), we obtain

$$
|\nabla p| \leqslant C(|\nabla y|+|\nabla m|) \quad \text { in } S
$$

and therefore

$$
|\Delta x|,|\Delta y|,|\Delta z|,|\Delta p|,|\Delta q| \leqslant C\left(|\nabla y|^{2}+|\nabla m|^{2}\right) \quad \text { in } S
$$

utilizing (3.27). In addition, formula (3.34) yields

$$
|\Delta m| \leqslant C\left(|\nabla y|^{2}+|\nabla m|^{2}\right) \quad \text { in } S
$$

because of $\left|\nabla_{\mathbf{y}}^{2} \psi(\mathbf{y}(x, y))\right| \leqslant C$ in $\bar{S}$. Consequently, the vector-valued function $\mathbf{x}(u, v):=$ $(y(u, v), m(u, v)) \in C^{2}\left(S, \mathbb{R}^{2}\right) \cap C^{0}\left(\bar{S}, \mathbb{R}^{2}\right)$ solves the system

$$
\begin{aligned}
& |\Delta \mathbf{x}(u, v)| \leqslant C|\nabla \mathbf{x}(u, v)|^{2}, \quad(u, v) \in S, \\
& \mathbf{x}(u, 0)=0, \quad u \in(-1,1) .
\end{aligned}
$$

Here we have additionally used the boundary condition (3.31) and the property (3.23) of the uniformizing map. With the aid of the Hilfssatz in [7] we see that there exists a $\varrho \in(0,1]$ such that $\mathbf{x} \in C^{1+\mu}\left(\overline{S_{\varrho}}\right)$ is valid for any $\mu \in(0,1)$. Now, we infer from (3.37), (3.22) and the integrability conditions

$$
f(u, v), z(u, v), p(u, v), q(u, v) \in C^{1+\mu}\left(\overline{S_{\varrho}}\right) .
$$

Combining potential theoretic results with (3.34), we may derive

$$
f, z, p, q \in C^{2+\alpha}\left(\overline{S_{\varrho / 4}}\right)
$$

as in the first part of the proof.

(3) Now, we have to go back to the original coordinates $(x, y)$. We calculate 


$$
\begin{aligned}
& z_{x x}(x, y)=\left.\frac{1}{J_{f}}\left(p_{u} y_{v}-p_{v} y_{u}\right)\right|_{f^{-1}(x, y)}, \\
& z_{x y}(x, y)=\left.\frac{1}{J_{f}}\left(-p_{u} x_{v}+p_{v} x_{u}\right)\right|_{f^{-1}(x, y)}, \\
& z_{y y}(x, y)=\left.\frac{1}{J_{f}}\left(-q_{u} x_{v}+q_{v} x_{u}\right)\right|_{f^{-1}(x, y)}, \quad(x, y) \in S .
\end{aligned}
$$

Consequently, we have to show $J_{f}(0,0)>0$. Then we obtain $z(x, y) \in C^{3+\alpha}\left(\overline{S_{r}}\right)$ with a sufficiently small $r>0$, using either (3.30) or (3.41).

At first, differentiating the first equation in (3.22) with respect to $v$ and the second equation with respect to $u$, a subsequent subtraction of the resulting equations yield

$$
\Delta y=\frac{c}{\sqrt{\Sigma}}\left\{\nabla_{\mathbf{z}}\left(\frac{b}{c}\right) \cdot\left(y_{v} \mathbf{z}_{u}-y_{u} \mathbf{z}_{v}\right)-\nabla_{\mathbf{z}}\left(\frac{\sqrt{\Sigma}}{c}\right) \cdot\left(y_{u} \mathbf{z}_{u}+y_{v} \mathbf{z}_{v}\right)\right\} \quad \text { in } S .
$$

Taking either (3.30) or (3.41) into account, we get the estimate

$$
\Delta y(u, v) \leqslant C|\nabla y(u, v)|, \quad(u, v) \in S_{\varrho / 4} .
$$

Combining this with (3.23), E. Hopf's maximum principle (compare e.g. [1], Section 4.6.4) yields

$$
y_{v}(0,0)>0
$$

and we infer $J_{f}(0,0)>0$ because of (3.25).

Now, we know that the solution of the transformed problem satisfies $z(x, y) \in$ $C^{3+\alpha}\left(\overline{S_{r}}\right)$. Reversing this transformation, we obtain that the solution of the original problem belongs to the class $C^{3+\alpha}\left(\bar{\Omega} \cap B_{\varepsilon}\left(x_{0}, y_{0}\right), \mathbb{R}\right)$ with a sufficiently small $\varepsilon>0$. Since the choice of $\left(x_{0}, y_{0}\right) \in \Gamma$ was arbitrarily, we finally conclude $z(x, y) \in C^{3+\alpha}(\Omega \cup$ $\Gamma, \mathbb{R})$.

THEOREM 2. - Let $z=z(x, y) \in C^{2}(\Omega, \mathbb{R}) \cap C^{1}(\Omega \cup \Gamma, \mathbb{R})$ be a solution for the quasilinear equation (3.1), and one of the boundary conditions (3.4), (3.5) is satisfied on the open, real analytic arc $\Gamma \subset \partial \Omega$. The functions $a, b, c, d: \Theta \rightarrow \mathbb{R}$ as well as $\varphi: \Gamma \rightarrow \mathbb{R}$ or $\psi: \mathcal{U} \rightarrow \mathbb{R}$ are real analytic in their domains of definition, and the ellipticity condition (3.6) is valid.

Then there exists a real analytic continuation of z across $\Gamma$, which solves Eq. (3.1) in the extended domain.

Proof. -

(1) In the first part, we study the Dirichlet boundary condition (3.4). Due to Lemma 3 we know $z \in C^{3}(\Omega \cup \Gamma, \mathbb{R})$. We only consider the case $\Omega=S, \Gamma=I$, and the homogeneous boundary condition (3.20). Inserting isothermal parameters $(u, v)$, we infer (3.21) or equivalently (3.22). Furthermore, the vector-valued function $\mathbf{z}=$ $(x, y, z, p, q) \in C^{2}(\bar{S})$ solves the system

$$
\Delta \mathbf{z}(u, v)=\mathbf{h}\left(\mathbf{z}(u, v), \mathbf{z}_{u}(u, v), \mathbf{z}_{v}(u, v)\right), \quad(u, v) \in S,
$$


with a real analytic right hand side $\mathbf{h}: \Theta_{0} \times \mathbb{R}^{5} \times \mathbb{R}^{5} \rightarrow \mathbb{R}^{5}, \Theta_{0}=\{\mathbf{z} \in \Theta \mid \Sigma(\mathbf{z})>0\}$. Now, we separate $\mathbf{z}=(\mathbf{x}, \mathbf{y})$ as follows,

$$
\mathbf{x}:=(y(u, v), z(u, v), p(u, v)), \quad \mathbf{y}:=(x(u, v), q(u, v)) .
$$

From the boundary condition (3.20) and the property (3.23) of the uniformizing map $f=(x(u, v), y(u, v)): \bar{S} \rightarrow \bar{S}$ we deduce

$$
\mathbf{x}(u, 0)=0, \quad u \in(-1,1) .
$$

The second equation in (3.22) yields

$$
x_{v}(u, 0)=\frac{b}{c} y_{v}(u, 0), \quad u \in(-1,1),
$$

on account of (3.44), and from the fourth equation we derive

$$
q_{v}(u, 0)=-\frac{d}{c} y_{v}(u, 0)-\frac{b}{c} p_{v}(u, 0), \quad u \in(-1,1) .
$$

Defining the real analytic matrix-valued function

$$
\mathbf{A}(\mathbf{y}):=\frac{1}{c(0, \mathbf{y})}\left(\begin{array}{ccc}
b(0, \mathbf{y}) & 0 & 0 \\
-d(0, \mathbf{y}) & 0 & -b(0, \mathbf{y})
\end{array}\right), \quad \mathbf{y} \in \mathcal{U}_{2},
$$

where $\mathcal{U}_{2} \subset \mathbb{R}^{2}$ is the projection of $\Theta_{0}$ onto the $x, q$-plane, we may rewrite the last two equations as

$$
\mathbf{y}_{v}(u, 0)=\mathbf{A}(\mathbf{y}(u, 0)) \cdot \mathbf{x}_{v}(u, 0), \quad u \in(-1,1) .
$$

With (3.43)-(3.45) we have found a boundary problem of the form considered in [13] Theorem 3. All the assumptions are fulfilled (set $\mathcal{U}_{1}:=\Theta_{0} \times \mathbb{R}^{5} \times \mathbb{R}^{5}$ ). Thus, for any $\varrho \in(0,1)$ we find $r>0$ such that $\mathbf{z}$ can be continued as a real analytic solution of (3.43) onto the set $S \cup((-\varrho, \varrho) \times(-r, r))$.

As in part (3) of the proof of Lemma 3 we see $J_{f}(0,0)>0$. Therefore, the mapping $w=(u(x, y), v(x, y))=f^{-1}: B_{\delta}(0,0) \rightarrow \mathcal{B}$ exists and is real analytic. Here $f$ denotes the just now constructed continuation of the uniformizing map, and $\mathcal{B} \subset$ $(-\varrho, \varrho) \times(-r, r)$ is an open neighbourhood of the point $(u, v)=(0,0)$. The function $z(x, y)=z \circ w(x, y)$ exists and is real analytic in $S \cup B_{\delta}(0,0)$, and $z(x, y)$ coincides in $S$ with the originial solution of (3.1). Finally, the function

$h(x, y):=a\left(x, y, z(x, y), z_{x}(x, y), z_{y}(x, y)\right) z_{x x}(x, y)+2 b(\ldots) z_{x y}+c(\ldots) z_{y y}+d(\ldots)$

is real analytic in $S \cup B_{\delta}(0,0)$ and vanishes in the open set $S$. Consequently, we have $h(x, y) \equiv 0$ in $S \cup B_{\delta}(0,0)$ according to the identity theorem in several variables, and we conclude that $z$ solves Eq. (3.1) in $S \cup B_{\delta}(0,0)$. If we reverse the transformation outlined at the beginning of this paragraph, we may transfer this result to the general case of an arbitrary analytic boundary arc $\Gamma$. 
(2) Let us now consider the (already transformed) boundary condition (3.31). We know $z \in C^{3}(\bar{S})$ due to Lemma 3, and we introduce isothermal parameters $(u, v)$. As in part 2 of the proof of Lemma 3 we consider the auxiliary function $m=m(u, v) \in$ $C^{2}(\bar{S}, \mathbb{R})$, which is defined in (3.32). The vector-valued function $\mathbf{z}=(\mathbf{x}, \mathbf{y})$ with

$$
\mathbf{x}(u, v):=(y(u, v), m(u, v)), \quad \mathbf{y}(u, v):=(x(u, v), z(u, v), p(u, v))
$$

solves a system of the form (3.43) with a real analytic right hand side $\mathbf{h}$ (note that $\psi$ is also real analytic). Furthermore, we learn

$$
\mathbf{x}(u, 0)=0, \quad u \in(-1,1),
$$

from (3.23) and (3.31). The second equation in (3.22) yields

$$
x_{v}(u, 0)=\frac{b}{c} y_{v}(u, 0), \quad u \in(-1,1),
$$

and with the aid of the relation $z_{v}=p x_{v}+q y_{v}$ we obtain

$$
z_{v}(u, 0)=\left(p(u, 0) \frac{b}{c}+\psi\right) y_{v}(u, 0), \quad u \in(-1,1),
$$

on account of (3.46). Solving the linear system of Eqs. (3.35), (3.36) with respect to $p_{u}$ and $p_{v}$, we infer

$$
p_{v}=\frac{\sqrt{\Sigma}\left(\psi_{x} x_{u}+\psi_{z} z_{u}\right)-\left(b+c \psi_{p}\right)\left(m_{v}+\frac{d}{c} y_{v}+\psi_{x} x_{v}+\psi_{z} z_{v}\right)}{a+2 b \psi_{p}+c \psi_{p}^{2}} \quad \text { on } I,
$$

where we have also exploited (3.46). Now, from the first equation in (3.22) and from (3.46) we deduce

$$
\psi_{x} x_{u}+\psi_{z} z_{u}=\left(\psi_{x}+p \psi_{z}\right) x_{u}=\frac{\sqrt{\Sigma}}{c}\left(\psi_{x}+p \psi_{z}\right) y_{v} \quad \text { on } I .
$$

Putting this and the relations (3.47), (3.48) into the formula (3.49), we obtain

$$
\begin{aligned}
p_{v}(u, 0)= & \frac{\Sigma\left(\psi_{x}+p \psi_{z}\right)-\left(b+c \psi_{p}\right)\left\{d+b \psi_{x}+(p b+c \psi) \psi_{z}\right\}}{c\left(a+2 b \psi_{p}+c \psi_{p}^{2}\right)} y_{v}(u, 0) \\
& -\frac{b+c \psi_{p}}{a+2 b \psi_{p}+c \psi_{p}^{2}} m_{v}(u, 0) \\
= & : k_{1}(\mathbf{y}(u, 0)) y_{v}(u, 0)+k_{2}(\mathbf{y}(u, 0)) m_{v}(u, 0), \quad u \in(-1,1) .
\end{aligned}
$$

We remark that $k_{1}$ und $k_{2}$ are real analytic functions on some open set $\mathcal{U}_{2} \subset \mathbb{R}^{3}$, which includes the set $\{\mathbf{y}(u, v) \mid(u, v) \in \bar{S}\}$. Defining the real analytic function

$$
\mathbf{A}(\mathbf{y}):=\frac{1}{c(0, \mathbf{y})}\left(\begin{array}{cc}
b(0, \mathbf{y}) & 0 \\
p b(0, \mathbf{y})+c(0, \mathbf{y}) \psi(\mathbf{y}) & 0 \\
c(0, \mathbf{y}) k_{1}(\mathbf{y}) & c(0, \mathbf{y}) k_{2}(\mathbf{y})
\end{array}\right), \quad \mathbf{y} \in \mathcal{U}_{1},
$$


we may write Eqs. (3.47), (3.48) and (3.50) as

$$
\mathbf{y}_{v}(u, 0)=\mathbf{A}(\mathbf{y}(u, 0)) \cdot \mathbf{x}_{v}(u, 0), \quad u \in(-1,1) .
$$

The problem (3.43), (3.46), (3.52) has the form considered in [13] Theorem 3. Consequently, we can continue $\mathbf{z}$ as a real analytic solution of (3.43) onto $S \cup((-\varrho, \varrho) \times$ $(-r, r))$ with an arbitrary $\varrho \in(0,1)$ and a suitable $r \in(0,1)$. We define the continuation of the quantity $q(u, v)$ by formula (3.32). Now, as in the first part of the proof, we find a continuation $z(x, y)$ of the given solution for (3.1) onto $S \cup B_{\delta}(0,0)$ with an appropriate number $\delta>0$, and the case of a general real analytic boundary $\operatorname{arc} \Gamma$ is treated in the usual way.

Example 1. - The surface $\mathcal{S}$ of a fluid in equilibrium, which is spanned into a vertical tube with a cross section $\Omega \subset \mathbb{R}^{2}$ and which is under the influence of capillarity, is called equilibrium capillary surface. One may describe $\mathcal{S}$ by a function $z=z(x, y)$, which satisfies the quasilinear, elliptic differential equation

$$
\left(1+z_{y}^{2}\right) z_{x x}-2 z_{x} z_{y} z_{x y}+\left(1+z_{x}^{2}\right) z_{y y}=\kappa z\left(1+|\nabla z|^{2}\right)^{3 / 2} \quad \text { in } \Omega .
$$

In (3.53) $\kappa$ is a physical constant. If we denote by $\gamma \in[0, \pi / 2]$ the angle between $\mathcal{S}$ and the tube at a point $z(x, y),(x, y) \in \partial \Omega$, then $z$ fulfils (after certain simplifications) the following boundary condition

$$
\mathbf{n} \cdot \frac{\nabla z(x, y)}{\sqrt{1+|\nabla z(x, y)|^{2}}}=\cos \gamma, \quad(x, y) \in \partial \Omega .
$$

In the case $\gamma \in(\pi / 2, \pi]$ one has to consider the function $-z(x, y)$. The boundary angle $\gamma$ is constant. For a detailed discussion of this and similar problems, including existence and uniqueness of a solution for (3.53), (3.54), we refer the reader to the monograph [2].

Relation (3.54) may be written in the form

$$
z_{\mathbf{n}}(x, y)=\cos \gamma \sqrt{1+\left(z_{\mathbf{t}}(x, y)\right)^{2}+\left(z_{\mathbf{n}}(x, y)\right)^{2}}, \quad(x, y) \in \partial \Omega .
$$

This equation is solvable with respect to $z_{\mathbf{n}}$ if $\cos \gamma<1$ is true, i.e. the surface $\mathcal{S}$ does not cross the tube in a tangential direction. In that case, we obtain

$$
z_{\mathbf{n}}(x, y)=\cot \gamma \sqrt{1+\left(z_{\mathbf{t}}(x, y)\right)^{2}}, \quad(x, y) \in \partial \Omega .
$$

Formula (3.55) is a nontangential boundary condition of first order in the sense of (3.5). Since $\psi(\zeta):=\cot \gamma \sqrt{1+\zeta^{2}}$ is real analytic for all $\zeta \in \mathbb{R}$, we learn from Theorem 2 the following: A capillary surface $\mathcal{S}$, which can be represented by a function $z \in C^{2}(\Omega) \cap C^{1}(\bar{\Omega})$, is a real analytic surface. Furthermore, $\mathcal{S}$ may be continued as a capillary surface across the tube, if $\partial \Omega$ is a real analytic Jordan arc and if $\gamma>0$ is satisfied.

This result is a generalization of H. Lewy's result [11]. He showed that a parametrized minimal surface $\mathbf{z}: S \cup I \rightarrow \mathbb{R}^{3}$, which maps $I$ into a real analytic support surface, can be 
continued across $I$ as a minimal surface. If $\mathbf{z}$ is a graph over the $x, y$-plane, this question corresponds to the problem (3.53), (3.54) with $\kappa=0$ and $\gamma=\pi / 2$.

Example 2. - Let $\Omega=S$ and $\Gamma=I$. The function

$$
z(x, y)=\sqrt{y} \mathrm{e}^{-(1+\sqrt{2}) x} \in C^{\infty}(S) \cap C^{0}(S \cup I)
$$

solves Eq. (3.1) in $S$, where the coefficients are defined as follows

$$
\begin{aligned}
& a=a(x, q)=2 q \mathrm{e}^{(1+\sqrt{2}) x}, \\
& b=b(x, y, q)=4 y q \mathrm{e}^{(1+\sqrt{2}) x}, \\
& c=c(x, y, q)=8 y^{2} q \mathrm{e}^{(1+\sqrt{2}) x}+1, \\
& d=d(x, q)=2 q^{3} \mathrm{e}^{2(1+\sqrt{2}) x} .
\end{aligned}
$$

Moreover, we have the boundary condition

$$
z(x, 0)=0, \quad x \in(-1,1) .
$$

The functions $a, b, c, d$ are real analytic and we calculate

$$
a c-b^{2}=2 q \mathrm{e}^{(1+\sqrt{2}) x}=y^{-1 / 2}>1 \quad \text { in } S .
$$

Obviously, there does not exist a real analytic continuation of $z$ across $I$ as a solution for (3.1). This means, if we assume $z \in C^{0}(\Omega \cup \Gamma)$, Theorem 2 is not true.

\section{The fully nonlinear equation}

Now we investigate the more general equation

$$
F\left(x, y, z(x, y), z_{x}(x, y), z_{y}(x, y), z_{x x}(x, y), z_{x y}(x, y), z_{y y}(x, y)\right)=0, \quad(x, y) \in \Omega,
$$

with a function $F=F(x, y, z, p, q, r, s, t) \in C^{2+\alpha}(\Theta, \mathbb{R})$ for $\alpha \in(0,1)$. Here $\Theta \subset \mathbb{R}^{8}$ denotes an open set. The ellipticity condition appears in the form

$$
\left.\left.\Sigma\right|_{\left(x, y, z(x, y), \ldots, z_{y y}(x, y)\right)}:=F_{r} F_{t}-\left.\frac{1}{4} F_{s}^{2}\right|_{(x, y, z(x, y), \ldots, z y y}(x, y)\right)>0, \quad(x, y) \in \Omega,
$$

and the inclusion $\left\{\left(x, y, z(x, y), \ldots, z_{y y}(x, y)\right) \mid(x, y) \in \Omega\right\} \subset \Theta$ has to be satisfied for the given solution $z=z(x, y) \in C^{2}(\Omega, \mathbb{R})$.

LEMMA 4. - Let $z=z(x, y) \in C^{2}(\Omega, \mathbb{R})$ be a solution of $(4.1)$ and let $\left(x_{0}, y_{0}\right) \in \Omega$, $r>0$ with $B_{r}\left(x_{0}, y_{0}\right) \subset \subset \Omega$. We may introduce isothermal parameters $(u, v) \in \bar{B}$ with respect to the Riemannian metric

$$
d s^{2}=F_{r} d y^{2}-F_{s} d x d y+F_{t} d x^{2} \in C^{1+\alpha}\left(\overline{B_{r}\left(x_{0}, y_{0}\right)}\right)
$$


with $F_{r}=F_{r}\left(x, y, z(x, y), \ldots, z_{y y}(x, y)\right)$ etc. Writing $z(u, v):=z(x(u, v), y(u, v))$ and $p=z_{x}, \ldots, t=z_{y y}$, the following relations are fulfilled

$$
\begin{aligned}
& y_{u}-\frac{1}{2} \frac{F_{s}}{F_{r}} x_{u}+\frac{\sqrt{\Sigma}}{F_{r}} x_{v}=0, \\
& y_{v}-\frac{\sqrt{\Sigma}}{F_{r}} x_{u}-\frac{1}{2} \frac{F_{s}}{F_{r}} x_{v}=0, \\
& r_{u}+\frac{1}{2} \frac{F_{s}}{F_{r}} s_{u}+\frac{\sqrt{\Sigma}}{F_{r}} s_{v}+\frac{D_{1}}{F_{r}} x_{u}=0, \\
& r_{v}-\frac{\sqrt{\Sigma}}{F_{r}} s_{u}+\frac{1}{2} \frac{F_{s}}{F_{r}} s_{v}+\frac{D_{1}}{F_{r}} x_{v}=0, \\
& t_{u}+\frac{1}{2} \frac{F_{s}}{F_{t}} s_{u}-\frac{\sqrt{\Sigma}}{F_{t}} s_{v}+\frac{D_{2}}{F_{t}} y_{u}=0, \\
& t_{v}+\frac{\sqrt{\Sigma}}{F_{t}} s_{u}+\frac{1}{2} \frac{F_{s}}{F_{t}} s_{v}+\frac{D_{2}}{F_{t}} y_{v}=0 \quad \text { in } B .
\end{aligned}
$$

Here we have abbreviated

$$
\begin{aligned}
& D_{1}(x, \ldots, t):=F_{x}+p F_{z}+r F_{p}+s F_{q}, \\
& D_{2}(x, \ldots, t):=F_{y}+q F_{z}+s F_{p}+t F_{q}, \quad(x, \ldots, t) \in \Theta .
\end{aligned}
$$

Moreover, the vector-valued function

$$
\begin{aligned}
\mathbf{z}(u, v):= & (x(u, v), y(u, v), z(u, v), p(u, v), q(u, v), r(u, v), s(u, v), t(u, v)) \\
& \in C^{2}\left(\bar{B}, \mathbb{R}^{8}\right)
\end{aligned}
$$

is a solution of the system

$$
\Delta \mathbf{z}(u, v)=\mathbf{h}\left(\mathbf{z}(u, v), \mathbf{z}_{u}(u, v), \mathbf{z}_{v}(u, v)\right), \quad(u, v) \in B,
$$

with a right hand side $\mathbf{h}: \Theta_{0} \times \mathbb{R}^{8} \times \mathbb{R}^{8} \rightarrow \mathbb{R}^{8} \in C^{\alpha}\left(\Theta_{0} \times \mathbb{R}^{8} \times \mathbb{R}^{8}, \mathbb{R}^{8}\right)$, which is defined on $\Theta_{0}:=\{\mathbf{z} \in \Theta \mid \Sigma(\mathbf{z})>0\}$ and whose exact form is given in the proof. Finally, if $F$ is a real analytic function in $\Theta$, then $\mathbf{h}$ is real analytic in $\Theta_{0} \times \mathbb{R}^{8} \times \mathbb{R}^{8}$.

Proof. -

(1) First we have $d s^{2} \in C^{1+\alpha}\left(\overline{B_{r}\left(x_{0}, y_{0}\right)}\right)$, since we obtain $z \in C^{4}(\Omega)$ from $F \in$ $C^{2+\alpha}(\Theta)$ due to [9] and [14]. The uniformizing map $f=(x(u, v), y(u, v)): \bar{B} \rightarrow$ $\overline{B_{r}\left(x_{0}, y_{0}\right)} \in C^{2+\alpha}\left(\bar{B}, \mathbb{R}^{2}\right)$ is homeomorphic and solves the Beltrami system

$$
\begin{aligned}
& y_{u}-\frac{1}{2} \frac{F_{s}}{F_{r}} x_{u}+\frac{\sqrt{\Sigma}}{F_{r}} x_{v}=0, \\
& y_{v}-\frac{\sqrt{\Sigma}}{F_{r}} x_{u}-\frac{1}{2} \frac{F_{s}}{F_{r}} x_{v}=0 \quad \text { in } B
\end{aligned}
$$


or equivalently

$$
\begin{aligned}
& x_{u}-\frac{1}{2} \frac{F_{s}}{F_{t}} y_{u}-\frac{\sqrt{\Sigma}}{F_{t}} y_{v}=0, \\
& x_{v}+\frac{\sqrt{\Sigma}}{F_{t}} y_{u}-\frac{1}{2} \frac{F_{s}}{F_{t}} y_{v}=0 \quad \text { in } B
\end{aligned}
$$

(cf. proof of Lemma 1). In order to derive the remaining four Equations in (4.4), we differentiate the equation $F=0$ with respect to $x$ and $y$. Then, we have

$$
\begin{aligned}
& F_{r} r_{x}+F_{s} s_{x}+F_{t} t_{x}+D_{1}=0, \\
& F_{r} r_{y}+F_{s} s_{y}+F_{t} t_{y}+D_{2}=0, \quad(x, y) \in B_{r}\left(x_{0}, y_{0}\right),
\end{aligned}
$$

using the abbreviations (4.5). From the first relations in (4.8), (4.9) and (4.10) we deduce

$$
\begin{aligned}
F_{r} r_{u} & =F_{r} r_{x} x_{u}+F_{r} r_{y} y_{u} \\
& =\left(-F_{s} s_{x}-F_{t} t_{x}-D_{1}\right) x_{u}+F_{r} s_{x} y_{u} \\
& =s_{x}\left(-F_{s} x_{u}+F_{r} y_{u}\right)-t_{x}\left(F_{t} x_{u}\right)-D_{1} x_{u} \\
& =s_{x}\left(-\frac{1}{2} F_{s} x_{u}-\sqrt{\Sigma} x_{v}\right)-s_{y}\left(\frac{1}{2} F_{s} y_{u}+\sqrt{\Sigma} y_{v}\right)-D_{1} x_{u} \\
& =-\frac{1}{2} F_{s} s_{u}-\sqrt{\Sigma} s_{v}-D_{1} x_{u}
\end{aligned}
$$

and consequently

$$
r_{u}+\frac{1}{2} \frac{F_{s}}{F_{r}} s_{u}+\frac{\sqrt{\Sigma}}{F_{r}} s_{v}+\frac{D_{1}}{F_{r}} x_{u}=0, \quad(u, v) \in B .
$$

Combining the first equation in (4.10) with the second relations in (4.8) and (4.9), we may calculate

$$
\begin{aligned}
F_{r} r_{v} & =F_{r} r_{x} x_{v}+F_{r} r_{y} y_{v} \\
& =\left(-F_{s} s_{x}-F_{t} t_{x}-D_{1}\right) x_{v}+F_{r} s_{x} y_{v} \\
& =s_{x}\left(-F_{s} x_{v}+F_{r} y_{v}\right)-t_{x}\left(F_{t} x_{v}\right)-D_{1} x_{v} \\
& =s_{x}\left(-\frac{1}{2} F_{s} x_{v}+\sqrt{\Sigma} x_{u}\right)-s_{y}\left(-\sqrt{\Sigma} y_{u}+\frac{1}{2} F_{s} y_{v}\right)-D_{1} x_{v} \\
& =\sqrt{\Sigma} s_{u}-\frac{1}{2} F_{s} s_{v}-D_{1} x_{v},
\end{aligned}
$$

which we write as

$$
r_{v}-\frac{\sqrt{\Sigma}}{F_{r}} s_{u}+\frac{1}{2} \frac{F_{s}}{F_{r}} s_{v}+\frac{D_{1}}{F_{r}} x_{v}=0, \quad(u, v) \in B .
$$


Finally, if we start with the second equation in (4.10) and proceed as above, we obtain

$$
t_{u}+\frac{1}{2} \frac{F_{s}}{F_{t}} s_{u}-\frac{\sqrt{\Sigma}}{F_{t}} s_{v}+\frac{D_{2}}{F_{t}} y_{u}=0, \quad(u, v) \in B
$$

and

$$
t_{v}+\frac{\sqrt{\Sigma}}{F_{t}} s_{u}+\frac{1}{2} \frac{F_{s}}{F_{t}} s_{v}+\frac{D_{2}}{F_{t}} y_{v}=0, \quad(u, v) \in B .
$$

(2) Now, we derive the system (4.7). Differentiating the first equation in (4.8) with respect to $u$ and the second one with respect to $v$ and summing up the resulting equations, we get

$$
\frac{1}{2} \frac{F_{s}}{F_{r}} \Delta x-\Delta y=-\frac{1}{2} \nabla_{\mathbf{z}}\left(\frac{F_{s}}{F_{r}}\right) \cdot\left(x_{u} \mathbf{z}_{u}+x_{v} \mathbf{z}_{v}\right)+\nabla_{\mathbf{z}}\left(\frac{\sqrt{\Sigma}}{F_{r}}\right) \cdot\left(x_{v} \mathbf{z}_{u}-x_{u} \mathbf{z}_{v}\right) \quad \text { in } B .
$$

Here we have written $\nabla_{\mathbf{z}}:=\left(\frac{\partial}{\partial x}, \ldots, \frac{\partial}{\partial t}\right)$. Next, if we differentiate the first equation in (4.8) with respect to $v$ and the second one with respect to $u$ and subtract the resulting equations from each other, we conclude

$$
\frac{\sqrt{\Sigma}}{F_{r}} \Delta x=-\frac{1}{2} \nabla_{\mathbf{z}}\left(\frac{F_{s}}{F_{r}}\right) \cdot\left(x_{v} \mathbf{z}_{u}-x_{u} \mathbf{z}_{v}\right)-\nabla_{\mathbf{z}}\left(\frac{\sqrt{\Sigma}}{F_{r}}\right) \cdot\left(x_{u} \mathbf{z}_{u}+x_{v} \mathbf{z}_{v}\right) \text { in } B .
$$

In the same way we obtain the relations

$$
\begin{aligned}
\frac{D_{1}}{F_{r}} \Delta x+\Delta r+\frac{1}{2} \frac{F_{s}}{F_{r}} \Delta s= & -\frac{1}{2} \nabla_{\mathbf{z}}\left(\frac{F_{s}}{F_{r}}\right) \cdot\left(s_{u} \mathbf{z}_{u}+s_{v} \mathbf{z}_{v}\right)-\nabla_{\mathbf{z}}\left(\frac{\sqrt{\Sigma}}{F_{r}}\right) \cdot\left(s_{v} \mathbf{z}_{u}-s_{u} \mathbf{z}_{v}\right) \\
& -\nabla_{\mathbf{z}}\left(\frac{D_{1}}{F_{r}}\right) \cdot\left(x_{u} \mathbf{z}_{u}+x_{v} \mathbf{z}_{v}\right) \quad \text { in } B
\end{aligned}
$$

and

$$
\begin{aligned}
\frac{\sqrt{\Sigma}}{F_{r}} \Delta s= & \frac{1}{2} \nabla_{\mathbf{z}}\left(\frac{F_{s}}{F_{r}}\right) \cdot\left(s_{v} \mathbf{z}_{u}-s_{u} \mathbf{z}_{v}\right)-\nabla_{\mathbf{z}}\left(\frac{\sqrt{\Sigma}}{F_{r}}\right) \cdot\left(s_{u} \mathbf{z}_{u}+s_{v} \mathbf{z}_{v}\right) \\
& +\nabla_{\mathbf{z}}\left(\frac{D_{1}}{F_{r}}\right) \cdot\left(x_{v} \mathbf{z}_{u}-x_{u} \mathbf{z}_{v}\right) \text { in } B
\end{aligned}
$$

from (4.11) and (4.12). Furthermore, Eqs. (4.13) and (4.14) yield

$$
\begin{aligned}
\frac{D_{2}}{F_{t}} \Delta y+\frac{1}{2} \frac{F_{s}}{F_{t}} \Delta s+\Delta t= & -\frac{1}{2} \nabla_{\mathbf{z}}\left(\frac{F_{s}}{F_{t}}\right) \cdot\left(s_{u} \mathbf{z}_{u}+s_{v} \mathbf{z}_{v}\right)+\nabla_{\mathbf{z}}\left(\frac{\sqrt{\Sigma}}{F_{t}}\right) \cdot\left(s_{v} \mathbf{z}_{u}-s_{u} \mathbf{z}_{v}\right) \\
& -\nabla_{\mathbf{z}}\left(\frac{D_{2}}{F_{t}}\right) \cdot\left(y_{u} \mathbf{z}_{u}+y_{v} \mathbf{z}_{v}\right) \quad \text { in } B,
\end{aligned}
$$


and the integrability conditions

$$
\begin{array}{ll}
z_{u}-p x_{u}-q y_{u}=0, & z_{v}-p x_{v}-q y_{v}=0, \\
p_{u}-r x_{u}-s y_{u}=0, & p_{v}-r x_{v}-s y_{v}=0, \\
q_{u}-s x_{u}-t y_{u}=0, & q_{v}-s x_{v}-t y_{v}=0 \quad \text { in } B
\end{array}
$$

give the relations

$$
\begin{aligned}
& -p \Delta x-q \Delta y+\Delta z=x_{u} p_{u}+x_{v} p_{v}+y_{u} q_{u}+y_{v} q_{v}, \\
& -r \Delta x-s \Delta y+\Delta p=x_{u} r_{u}+x_{v} r_{v}+y_{u} s_{u}+y_{v} s_{v}, \\
& -s \Delta x-t \Delta y+\Delta q=x_{u} s_{u}+x_{v} s_{v}+y_{u} t_{u}+y_{v} t_{v} \text { in } B .
\end{aligned}
$$

We interpret the eight Eqs. (4.15)-(4.22) as a linear system for the eight unknowns $\Delta x, \ldots, \Delta t$. Calculating

$$
\operatorname{det}(\text { matrix of coefficients })=-\frac{\Sigma}{F_{r}^{2}}<0 \quad \text { in } B,
$$

we solve this system. If we write $m_{1}, \ldots, m_{8}$ for the right hand sides of (4.15)-(4.22), we obtain the following semilinear system

$$
\begin{aligned}
& \Delta x=\frac{F_{r}}{\sqrt{\Sigma}} m_{2}, \\
& \Delta y=-m_{1}+\frac{1}{2} \frac{F_{s}}{\sqrt{\Sigma}} m_{2}, \\
& \Delta z=-q m_{1}+\frac{1}{\sqrt{\Sigma}}\left(p F_{r}+\frac{1}{2} q F_{s}\right) m_{2}+m_{6}, \\
& \Delta p=-s m_{1}+\frac{1}{\sqrt{\Sigma}}\left(r F_{r}+\frac{1}{2} s F_{s}\right) m_{2}+m_{7}, \\
& \Delta q=-t m_{1}+\frac{1}{\sqrt{\Sigma}}\left(s F_{r}+\frac{1}{2} t F_{s}\right) m_{2}+m_{8}, \\
& \Delta r=-\frac{D_{1}}{\sqrt{\Sigma}} m_{2}+m_{3}-\frac{1}{2} \frac{F_{s}}{\sqrt{\Sigma}} m_{4}, \\
& \Delta s=\frac{F_{r}}{\sqrt{\Sigma}} m_{4}, \\
& \Delta t=\frac{D_{2}}{F_{t}}\left(m_{1}-\frac{1}{2} \frac{F_{s}}{\sqrt{\Sigma}} m_{2}\right)-\frac{1}{2} \frac{F_{r}}{F_{t}} \frac{F_{s}}{\sqrt{\Sigma}} m_{4}+m_{5} \quad \text { in } B .
\end{aligned}
$$

Now, using Lemma 4 and following the proof of Theorem 1, we infer

THEOREM 3 (Bernstein's analyticity theorem). - Any solution $z=z(x, y) \in C^{2}(\Omega$, $\mathbb{R})$ of (4.1)-(4.2) is real analytic in $\Omega$, if $F$ is a real analytic function in $\Theta$.

Next we investigate the boundary behaviour for solutions $z=z(x, y) \in C^{2}(\Omega \cup \Gamma, \mathbb{R})$ of (4.1), where $\Gamma \subset \partial \Omega \neq \emptyset$ denotes an open Jordan arc as described at the beginning 
of paragraph 3. Again we study either Dirichlet data (3.4) or nontangential data of first order (3.5). Supposing $\left\{\left(x, y, z(x, y), \ldots, z_{y y}(x, y)\right) \mid(x, y) \in \Omega \cup \Gamma\right\}$, we replace the ellipticity condition (4.2) by

$$
\left.\left.\Sigma\right|_{\left(x, y, z(x, y), \ldots, z_{y y}(x, y)\right)}:=F_{r} F_{t}-\left.\frac{1}{4} F_{s}^{2}\right|_{(x, y, z(x, y), \ldots, z y y}(x, y)\right)>0, \quad(x, y) \in \Omega \cup \Gamma .
$$

As in the case of quasilinear equations, it suffices to consider the case $\Omega=S, \Gamma=I$, applying a local transformation. Moreover, we study homogeneous Dirichlet data

$$
z(x, 0)=0, \quad x \in(-1,1),
$$

subtracting a continuation of the boundary function $\varphi$ from the given solution. The boundary condition (3.5) appears in the form

$$
z_{y}(x, 0)=\psi\left(x, z(x, 0), z_{x}(x, 0)\right), \quad x \in(-1,1),
$$

where $\psi: \mathcal{U} \rightarrow \mathbb{R}$ is defined on an open set $\mathcal{U} \subset \mathbb{R}^{3}$ with $\left\{\left(x, z(x, y), z_{x}(x, y)\right) \mid(x, y) \in\right.$ $\bar{S}\} \subset \mathcal{U}$.

Lemma 5. - Let $z=z(x, y) \in C^{2}(\Omega \cup \Gamma, \mathbb{R})$ be a solution of (4.1) with $F \in$ $C^{2+\alpha}(\Theta, \mathbb{R}), \alpha \in(0,1)$, and the ellipticity property (4.24) is satisfied. On the $C^{4+\alpha}$-arc $\Gamma$ we assume either (3.4) or (3.5) and we suppose $\varphi \in C^{4+\alpha}(\Gamma, \mathbb{R})$ and $\psi \in C^{3+\alpha}(\mathcal{U}, \mathbb{R})$, respectively. Then we have $z \in C^{4+\alpha}(\Omega \cup \Gamma, \mathbb{R})$.

\section{Proof. -}

(1) Due to the results of Hopf [9] and Nirenberg [14] we know $z \in C^{4+\alpha}(\Omega, \mathbb{R})$. We suppose $\Omega=S, \Gamma=I$ and consider the homogeneous Dirichlet condition (4.25). Introducing isothermal parameters $(u, v) \in \bar{S}$, we deduce the system (4.7) for $\mathbf{z}=$ $(x(u, v), \ldots, t(u, v)) \in C^{2+\alpha}(S) \cap C^{0}(\bar{S})$. The uniformizing map $f: \bar{S} \rightarrow \bar{S}$ may be chosen such that (3.23) is satisfied. From the proof of Lemma 4 we take the exact shape of the right hand side in (4.7), and we obtain estimates of the form

$$
|\Delta x|, \ldots,|\Delta t| \leqslant C\left(|\nabla x|^{2}+\cdots+|\nabla t|^{2}\right) \quad \text { in } S
$$

exploiting condition (4.24) (or more precisely, its transformed version). Furthermore, we know the system (4.4) to be fulfilled. The first two equations in (4.4) yield

$$
|\nabla x|^{2}=\frac{F_{r}}{F_{t}}|\nabla y|^{2} \quad \text { in } S,
$$

and together with the integrability conditions we conclude

$$
|\nabla x|,|\nabla z|,|\nabla p|,|\nabla q| \leqslant C|\nabla y| \quad \text { in } S \text {. }
$$


The third and the fourth equation in (4.4) are solvable with respect to $s_{u}$ and $s_{v}$. Utilizing the first two equations in (4.4), we get

$$
\begin{aligned}
& s_{u}+\frac{1}{2} \frac{F_{s}}{F_{t}} r_{u}-\frac{\sqrt{\Sigma}}{F_{t}} r_{v}+\frac{D_{1}}{F_{t}} y_{u}=0, \\
& s_{v}+\frac{\sqrt{\Sigma}}{F_{t}} r_{u}+\frac{1}{2} \frac{F_{s}}{F_{t}} r_{v}+\frac{D_{1}}{F_{t}} y_{v}=0 \text { in } S .
\end{aligned}
$$

These and the last two equations in (4.4) yield the following estimates

$$
|\nabla s|,|\nabla t| \leqslant C(|\nabla y|+|\nabla r|) \quad \text { in } S .
$$

Let us now consider the function $\mathbf{x}=(y, r) \in C^{2+\alpha}\left(S, \mathbb{R}^{2}\right) \cap C^{0}\left(\bar{S}, \mathbb{R}^{2}\right)$. Using $y(u, 0)=$ 0 and the boundary condition (4.25), we conclude

$$
\begin{aligned}
& |\Delta \mathbf{x}(u, v)| \leqslant C|\nabla \mathbf{x}(u, v)|^{2}, \quad(u, v) \in S, \\
& \mathbf{x}(u, 0)=0, \quad u \in(-1,1),
\end{aligned}
$$

from (4.27), (4.29) and (4.31). The Hilfssatz in [7] shows $\mathbf{x} \in C^{1+\mu}\left(\overline{S_{\varrho}}\right)$ with a sufficiently small $\varrho \in(0,1]$ and an arbitrary $\mu \in(0,1)$. We obtain $\mathbf{z} \in C^{1+\mu}\left(\overline{S_{\varrho}}\right)$ and some potential theory shows

$$
\mathbf{z} \in C^{2+\alpha}\left(\overline{S_{\varrho / 4}}\right) .
$$

As in the proof of Lemma 3 one sees $J_{f}(0,0)>0$, such that we infer $z(x, y) \in C^{4+\alpha}\left(\overline{S_{r}}\right)$ for a sufficiently small $r \in(0,1)$. In order to derive this last regularity property, one has to use relations as $z_{x x x}=J_{f}^{-1}\left(r_{u} y_{v}-r_{v} y_{u}\right)$.

(2) Let us now study the boundary condition (4.26). We define

$$
\chi(\mathbf{y})=\chi(x, z, p, r):=\psi_{x}(x, z, p)+\psi_{z}(x, z, p) p+\psi_{p}(x, z, p) r \in C^{2+\alpha}(\mathcal{U} \times \mathbb{R}, \mathbb{R})
$$

and introduce the auxiliary function

$$
n(u, v):=s(u, v)-\chi(\mathbf{y}(u, v)) \in C^{2+\alpha}(S) \cap C^{0}(\bar{S}) .
$$

From (4.26) and $y(u, 0)=0$ we infer

$$
n(u, 0)=0, \quad u \in(-1,1)
$$

Differentiating (4.34) and inserting the relations (4.30), we obtain

$$
\begin{aligned}
& n_{u}=-\left(\frac{1}{2} \frac{F_{s}}{F_{t}}+\psi_{p}\right) r_{u}+\frac{\sqrt{\Sigma}}{F_{t}} r_{v}-\frac{D_{1}}{F_{t}} y_{u}-\chi_{x} x_{u}-\chi_{z} z_{u}-\chi_{p} p_{u}, \\
& n_{v}=-\frac{\sqrt{\Sigma}}{F_{t}} r_{u}-\left(\frac{1}{2} \frac{F_{s}}{F_{t}}+\psi_{p}\right) r_{v}-\frac{D_{1}}{F_{t}} y_{v}-\chi_{x} x_{v}-\chi_{z} z_{v}-\chi_{p} p_{v} \text { in } S .
\end{aligned}
$$


Since we may solve (4.36) with respect to $r_{u}$ and $r_{v}$, we deduce

$$
|\nabla r| \leqslant C(|\nabla y|+|\nabla n|) \quad \text { in } S
$$

with the aid of (4.29). Putting this into (4.30), we also get

$$
|\nabla s| \leqslant C(|\nabla y|+|\nabla n|) \quad \text { in } S,
$$

and the last two equations in (4.4) yield

$$
|\nabla t| \leqslant C(|\nabla y|+|\nabla n|) \quad \text { in } S .
$$

Finally, from

$$
\Delta n=\Delta s-\nabla_{\mathbf{y}} \chi \cdot \Delta \mathbf{y}-\mathbf{y}_{u} \cdot \nabla_{\mathbf{y}}^{2} \chi \cdot \mathbf{y}_{u}-\mathbf{y}_{v} \cdot \nabla_{\mathbf{y}}^{2} \chi \cdot \mathbf{y}_{v}
$$

and the estimates (4.27), (4.29) as well as (4.37)-(4.39) we infer

$$
|\Delta y|,|\Delta n| \leqslant C\left(|\nabla y|^{2}+|\nabla n|^{2}\right) \quad \text { in } S .
$$

Here we have abbreviated $\nabla_{\mathbf{y}} \chi:=\left(\chi_{x}, \chi_{z}, \chi_{p}, \chi_{r}\right)$, and $\nabla_{\mathbf{y}}^{2} \chi$ denotes the Hessian matrix of $\chi$.

Because of (3.23), (4.35) and (4.40), the function $\mathbf{x}=(y, n) \in C^{2+\alpha}(S) \cap C^{0}(\bar{S})$ is a solution of the problem

$$
\begin{aligned}
& |\Delta \mathbf{x}(u, v)| \leqslant C|\nabla \mathbf{x}(u, v)|^{2}, \quad(u, v) \in S, \\
& \mathbf{x}(u, 0)=0, \quad u \in(-1,1),
\end{aligned}
$$

and therefore we have $\mathbf{x} \in C^{1+\mu}\left(\overline{S_{\varrho}}\right)$ for $\mu \in(0,1)$ and an appriorate $\varrho \in(0,1]$. Consequently, we infer $\mathbf{z} \in C^{1+\mu}\left(\overline{S_{\varrho}}\right)$ with the aid of (4.4) and (4.36). Using pontential theory, we get $\mathbf{z} \in C^{2+\alpha}\left(\overline{S_{\varrho / 4}}\right)$ and because of $J_{f}(0,0)>0$ we obtain $z=z(x, y) \in$ $\left.C^{4+\alpha} \overline{\left(S_{r}\right.}\right)$ for sufficiently small $r \in(0,1)$.

THEOREM 4. - A solution $z=z(x, y) \in C^{2}(\Omega \cup \Gamma, \mathbb{R})$ of the fully nonlinear equation (4.1) is given. The ellipticity condition (4.24) is valid, and $F$ is a real analytic function. On the real analytic Jordan arc $\Gamma \subset \partial \Omega$ one of the boundary conditions (3.4), (3.5) is satisfied, where $\varphi$ and $\psi$ respectively are real analytic functions on their domains of definition. Then we can continue $z$ as a real analytic solution for (4.1) across $\Gamma$.

Proof. -

(1) From Lemma 5 we learn $z \in C^{4}(\Omega \cup \Gamma)$. We consider the Dirichlet condition in the case $\Omega=S, \Gamma=I$, that is (4.25). The isothermally parametrized function $\mathbf{z}(u, v)=(x(u, v), \ldots, t(u, v)) \in C^{2}\left(\bar{S}, \mathbb{R}^{8}\right)$ solves the system

$$
\Delta \mathbf{z}(u, v)=\mathbf{h}\left(\mathbf{z}(u, v), \mathbf{z}_{u}(u, v), \mathbf{z}_{v}(u, v)\right), \quad(u, v) \in S,
$$


according to Lemma 4, and the right hand side $\mathbf{h}$ is real analytic in $\Theta_{0} \times \mathbb{R}^{8} \times \mathbb{R}^{8}$. Moreover, we know that the system of first order (4.4) is satisfied. We separate $\mathbf{z}=(\mathbf{x}, \mathbf{y})$ as follows,

$$
\mathbf{x}:=(y, z, p, r), \quad \mathbf{y}:=(x, q, s, t) .
$$

The boundary condition (4.25) and the property (3.23) of the uniformizing map $f=$ $(x, y)$ yield

$$
\mathbf{x}(u, 0)=0, \quad u \in(-1,1) .
$$

From the second equation in (4.4) we get

$$
x_{v}(u, 0)=\frac{1}{2} \frac{F_{s}}{F_{t}} y_{v}(u, 0), \quad u \in(-1,1)
$$

and together with $q_{v}=s x_{v}+t y_{v}$ we obtain

$$
q_{v}(u, 0)=\left(\frac{1}{2} \frac{F_{s}}{F_{t}} s+t\right) y_{v}(u, 0), \quad u \in(-1,1) .
$$

Solving the third and the fourth equation in (4.4) with respect to $s_{u}$ and $s_{v}$, we infer (4.30) and in particular

$$
s_{v}(u, 0)=-\frac{D_{1}}{F_{t}} y_{v}(u, 0)-\frac{1}{2} \frac{F_{s}}{F_{t}} r_{v}(u, 0), \quad u \in(-1,1),
$$

taking (4.43) into account. Finally, the last equation in (4.4) yields

$$
t_{v}(u, 0)=\frac{1}{F_{t}^{2}}\left(\frac{1}{2} F_{s} D_{1}-F_{t} D_{2}\right) y_{v}(u, 0)-\frac{1}{F_{t}^{2}}\left(\Sigma-\frac{1}{4} F_{s}^{2}\right) r_{v}(u, 0), \quad u \in(-1,1) .
$$

Here we have used (4.46) and the first equation in (4.30), which, on $I$, has the form

$$
s_{u}(u, 0)=\frac{\sqrt{\Sigma}}{F_{t}} r_{v}(u, 0), \quad u \in(-1,1) .
$$

Equations (4.44)-(4.47) may be written as

$$
\mathbf{y}_{v}(u, 0)=\mathbf{A}(\mathbf{y}(u, 0)) \cdot \mathbf{x}_{v}(u, 0), \quad u \in(-1,1),
$$

where $\mathbf{A}: \mathcal{U}_{2} \rightarrow \mathbb{R}^{4 \times 4}$ denotes a real analytic, matrix-valued function on the open set $\mathcal{U}_{2}$, which is the projection of $\Theta_{0}$ onto the $x, q, s, t$-subspace.

In (4.42), (4.43) and (4.48) we have found a system of the form considered in [13] Theorem 3. Now, proceeding as in the proof of Theorem 2, we find $\delta>0$ such that $z(x, y)=z \circ f^{-1}(x, y)$ exists and is a real analytic function in $S \cup B_{\delta}(0,0)$. Since

$$
h(x, y):=F\left(x, y, z(x, y), \ldots, z_{y y}(x, y)\right)
$$


is real analytic in $S \cup B_{\delta}(0,0)$ and vanishes in $S$, the identity theorem in several variables shows that $z(x, y)$ solves the equation (4.1) in $S \cup B_{\delta}(0,0)$.

(2) Let us now consider the boundary condition (4.26). With the real analytic function

$$
\chi(x, z, p, r):=\psi_{x}(x, z, p)+\psi_{z}(x, z, p) p+\psi_{p}(x, z, p) r, \quad(x, z, p, r) \in \mathcal{U} \times \mathbb{R},
$$

we consider the quantity $n=n(u, v) \in C^{2}(\bar{S})$ defined in (4.34). Setting

$$
\mathbf{x}:=(y, n), \quad \mathbf{y}:=(x, z, p, q, r, t),
$$

we obtain

$$
\mathbf{x}(u, 0)=0, \quad u \in(-1,1) .
$$

Using this and (4.44), the integrability conditions yield

$$
\begin{aligned}
z_{v}(u, 0) & =\left(\frac{1}{2} \frac{F_{s}}{F_{t}} p+q\right) y_{v}(u, 0), \\
p_{v}(u, 0) & =\left(\frac{1}{2} \frac{F_{s}}{F_{t}} r+\chi(x, z, p, r)\right) y_{v}(u, 0), \\
q_{v}(u, 0) & =\left(\frac{1}{2} \frac{F_{s}}{F_{t}} \chi(x, z, p, r)+t\right) y_{v}(u, 0), \quad u \in(-1,1) .
\end{aligned}
$$

Moreover, the first equation in (4.4) and the integrability conditions give

$$
\begin{aligned}
& x_{u}(u, 0)=\frac{\sqrt{\Sigma}}{F_{t}} y_{v}(u, 0), \\
& z_{u}(u, 0)=\frac{\sqrt{\Sigma}}{F_{t}} p y_{v}(u, 0), \\
& p_{u}(u, 0)=\frac{\sqrt{\Sigma}}{F_{t}} r y_{v}(u, 0), \\
& q_{u}(u, 0)=\frac{\sqrt{\Sigma}}{F_{t}} \chi(x, z, p, r) y_{v}(u, 0), \quad u \in(-1,1) .
\end{aligned}
$$

The function $n(u, v)$ satisfies (4.36). Since we may solve this system with respect to $r_{u}$ and $r_{v}$, we find real analytic functions $\mathbf{f}_{i}=\mathbf{f}_{i}(\mathbf{y}): \mathcal{U}_{2} \rightarrow \mathbb{R}^{2}, i=1,2$, such that

$$
\begin{aligned}
& r_{u}(u, 0)=\mathbf{f}_{1}(\mathbf{y}(u, 0)) \cdot \mathbf{x}_{v}(u, 0), \\
& r_{v}(u, 0)=\mathbf{f}_{2}(\mathbf{y}(u, 0)) \cdot \mathbf{x}_{v}(u, 0), \quad u \in(-1,1),
\end{aligned}
$$

holds true. In order to deduce this system, one has to utilize the relations (4.44), (4.50) and (4.51). The set $\mathcal{U}_{2}$ has the property $\{\mathbf{y}(u, v) \mid(u, v) \in \bar{S}\} \subset \mathcal{U}_{2}$.

Now, inserting (4.30) and (4.52)-(4.53) into the last equation of (4.4), we infer

$$
t_{v}(u, 0)=\mathbf{f}_{3}(\mathbf{y}(u, 0)) \cdot \mathbf{x}_{v}(u, 0), \quad u \in(-1,1),
$$


with a further real analytic function $\mathbf{f}_{3}=\mathbf{f}_{3}(\mathbf{y}): \mathcal{U}_{2} \rightarrow \mathbb{R}^{2}$. Eqs. (4.44), (4.50), (4.53) and (4.54) may be written as

$$
\mathbf{y}_{v}(u, 0)=\mathbf{A}(\mathbf{y}(u, 0)) \cdot \mathbf{x}_{v}(u, 0), \quad u \in(-1,1),
$$

where $\mathbf{A}=\mathbf{A}(\mathbf{y}): \mathcal{U}_{2} \rightarrow \mathbb{R}^{6 \times 2}$ denotes a real analytic, matrix-valued function. Finally, because of

$$
\Delta n=\Delta s-\nabla_{\mathbf{y}} \chi \cdot \Delta \mathbf{y}-\mathbf{y}_{u} \cdot \nabla_{\mathbf{y}}^{2} \chi \cdot \mathbf{y}_{u}-\mathbf{y}_{v} \cdot \nabla_{\mathbf{y}}^{2} \chi \cdot \mathbf{y}_{v},
$$

the function $\mathbf{z}=(\mathbf{x}, \mathbf{y})$ solves a system of the form (4.42).

Due to [13] Theorem 3, the solution $\mathbf{z}$ of the problem (4.42), (4.49), (4.55) can be continued as a real analytic solution for (4.42) across $I$, and we define the continuation of $s(u, v)$ by (4.34). Using $J_{f}(0,0)>0$, we find a continuation $z(x, y)=z \circ f^{-1}(x, y)$ of the original solution for (4.1) onto the set $S \cup B_{\delta}(0,0)(\delta>0$ sufficiently small). This $z$ is real analytic and solves (4.1) in its domain of definition.

Example 3. - The function $z(x, y)=y^{3 / 2} \mathrm{e}^{x} \in C^{2}(S) \cap C^{1}(S \cup I)$ is a solution of the equation (4.1) with

$$
F=F(x, q, r, t)=r t^{2}-\frac{3}{8} q \mathrm{e}^{2 x} .
$$

$z$ satisfies the boundary condition

$$
z(x, 0)=0, \quad x \in(-1,1),
$$

as well as

$$
z_{y}(x, 0)=0, \quad x \in(-1,1) .
$$

The function $F$ is real analytic and we have

$$
F_{r} F_{t}-\frac{1}{4} F_{s}^{2}=2 r t^{3}=\frac{27}{32} \mathrm{e}^{4 x}>0 \quad \text { in } S .
$$

But it is not possible to continue $z$ across $I$ as a real analytic solution of (4.1). Therefore, Theorem 4 is not true for solutions $z \in C^{2}(\Omega) \cap C^{1}(\Omega \cup \Gamma)$.

\section{REFERENCES}

[1] R. Courant, D. Hilbert, Methods of Mathematical Physics II, Wiley, New York, 1989.

[2] R. Finn, Equilibrium Capillary Surfaces, Springer, New York, 1986.

[3] A. Friedman, On the regularity of the solutions of nonlinear elliptic and parabolic systems of partial differential equations, J. Math. Mech. 7 (1958) 43-59.

[4] P.R. Garabedian, Partial Differential Equations, Chelsea, New York, 1989.

[5] H. Grauert, K. Fritzsche, Einführung in die Funktionentheorie mehrerer Veränderlicher, Springer, Berlin, 1974.

[6] D. Gilbarg, N.S. Trudinger, Elliptic Partial Differential Equations of Second Order, Springer, Berlin, 1983. 
[7] E. Heinz, Über das Randverhalten quasilinearer elliptischer Systeme mit isothermen Parametern, Math. Z. 113 (1970) 99-105.

[8] E. Heinz, Lokale Abschätzungen und Randverhalten von Lösungen elliptischer MongeAmpèrescher Gleichungen, J. Reine Angew. Math. 438 (1993) 1-29.

[9] E. Hopf, Über den funktionalen, insbesondere den analytischen Charakter der Lösungen elliptischer Differentialgleichungen zweiter Ordnung, Math. Z. 34 (1931) 194-233.

[10] H. Lewy, Neuer Beweis des analytischen Charakters der Lösungen elliptischer Differentialgleichungen, Math. Ann. 101 (1929) 609-619.

[11] H. Lewy, On minimal surfaces with partially free boundary, Comm. Pure Appl. Math. 4 (1951) 1-13.

[12] C.B. Morrey, Multiple Integrals in the Calculus of Variations, Springer, Berlin, 1966.

[13] F. Müller, Analyticity of solutions for semilinear elliptic systems of second order, Calc. Var. Partial Differentials Equuations, to appear.

[14] L. Nirenberg, On non-linear elliptic partial differential equations and Hölder continuity, Comm. Pure Appl. Math. 6 (1953) 103-156.

[15] F. Sauvigny, Introduction of isothermal parameters into a Riemannian metric by the continuity method, Analysis 19 (1999) 235-243.

[16] F. Schulz, Regularity Theory for Quasilinear Elliptic Systems Monge-Ampère Equations in Two Dimensions, Springer, Berlin, 1990. 